\title{
Monocyte dysregulation and systemic inflammation during pediatric falciparum malaria
}

\author{
Katherine R. Dobbs, ${ }^{1,2}$ Paula Embury, ${ }^{1}$ John Vulule, ${ }^{3}$ Peter S. Odada, ${ }^{3}$ Bruce A. Rosa, ${ }^{4}$ \\ Makedonka Mitreva, ${ }^{4}$ James W. Kazura, ${ }^{1}$ and Arlene E. Dent ${ }^{1,2}$ \\ 'Center for Global Health and Diseases, Case Western Reserve University, Cleveland, Ohio, USA. 'Division of Pediatric \\ Infectious Diseases, University Hospitals Rainbow Babies and Children's Hospital, Cleveland, Ohio, USA. ${ }^{3}$ Centre for Global \\ Health Research, Kenya Medical Research Institute, Kisumu, Kenya. ${ }^{4}$ McDonnell Genome Institute, Washington University \\ School of Medicine, St. Louis, Missouri, USA.
}

BACKGROUND. Inflammation and monocytes are thought to be important to human malaria pathogenesis. However, the relationship of inflammation and various monocyte functions to acute malaria, recovery from acute malaria, and asymptomatic parasitemia in endemic populations is poorly understood.

METHODS. We evaluated plasma cytokine levels, monocyte subsets, monocyte functional responses, and monocyte inflammatory transcriptional profiles of 1- to 10-year-old Kenyan children at the time of presentation with acute uncomplicated malaria and at recovery 6 weeks later; these results were compared with analogous data from asymptomatic children and adults in the same community.

RESULTS. Acute malaria was marked by elevated levels of proinflammatory and regulatory cytokines and expansion of the inflammatory "intermediate" monocyte subset that returned to levels of healthy asymptomatic children 6 weeks later. Monocytes displayed activated phenotypes during acute malaria, with changes in surface expression of markers important to innate and adaptive immunity. Functionally, acute malaria monocytes and monocytes from asymptomatic infected children had impaired phagocytosis of $P$. falciparum-infected erythrocytes relative to asymptomatic children with no blood-stage infection. Monocytes from both acute malaria and recovery time points displayed strong and equivalent cytokine responsiveness to innate immune agonists that were independent of infection status. Monocyte transcriptional profiles revealed regulated and balanced proinflammatory and antiinflammatory and altered phagocytosis gene expression patterns distinct from malaria-naive monocytes.

CONCLUSION. These observations provide insights into monocyte functions and the innate immune response during uncomplicated malaria and suggest that asymptomatic parasitemia in children is not clinically benign.

FUNDING. Support for this work was provided by NIH/National Institute of Allergy and Infectious Diseases (R01AI095192-05), the Burroughs Wellcome Fund/American Society of Tropical Medicine and Hygiene, and the Rainbow Babies \& Children's Foundation.

Conflict of interest: The authors have declared that no conflict of interest exists.

Submitted: May 24, 2017

Accepted: August 16, 2017

Published: September 21, 2017

Reference information:

JCI Insight. 2017;2(18):e95352.

https://doi.org/10.1172/jci.

insight. 95352

\section{Introduction}

Malaria continues to pose an enormous health problem worldwide, with approximately 200 million new cases annually (1). In endemic areas, blood-stage Plasmodium falciparum infection of children results in a spectrum of clinical findings, from asymptomatic parasitemia to uncomplicated malaria to life-threatening severe malaria and death (2). Compared with adults and adolescents, young children living in endemic areas where transmission is stable and relatively intense are susceptible to more frequent symptomatic infections and severe disease, and, despite decreasing transmission in some areas, the burden of malaria in young children in sub-Saharan Africa remains substantial $(3,4)$. The mechanisms by which this age-related naturally acquired immunity to clinical malaria is generated and maintained are poorly understood (5). 
Cells of the monocyte/macrophage lineage play a key role at the interface between innate and adaptive immunity and are thought to be essential for host protection against malaria (6). Monocytes perform crucial effector functions in host defense against $P$. falciparum infection, including nonopsonic and opsonic phagocytosis of $P$. falciparum-infected erythrocytes (IEs) (7-11). Monocyte interactions with products of the parasite itself, e.g., hemozoin and glycophosphatidylinositols (12), and erythrocyte byproducts resulting from hemolysis, e.g., extracellular heme (13), by innate immune receptors, such as TLRs, have been observed to affect downstream inflammatory cytokine production (14-16) and modulate adaptive immune responses $(17,18)$. While activation of the innate immune response is necessary to control blood-stage infection, excessive activation of monocytes and other innate immune cells promotes disease pathogenesis. Similar to sepsis, monocyte release of proinflammatory cytokines, such as IL-1 $\beta$ and TNF, during acute malaria augments systemic inflammation and is associated with signs of severe malaria pathogenesis, e.g., lactic acidosis, hypoglycemia, respiratory distress (19). In severe malaria anemia, monocytes not only control infection by phagocytosing infected and uninfected erythrocytes, but also produce inflammatory mediators, such as IL-6, NO, and TNF, that impair erythropoiesis (20). Better characterization of the changes in monocyte subpopulations and their functions during symptomatic and asymptomatic infection would aid in determining the factors that underlie the balance between immune protection and immunopathology.

Circulating human monocytes are a heterogeneous population that has been classified into three subsets based on levels of expression of CD14 (LPS coreceptor) and CD16 (the low-affinity Fc $\gamma$ receptor III [Fc $\gamma$ RIII]). The subsets include CD $14^{++} \mathrm{CD} 16^{-}$"classical," CD $14^{++} \mathrm{CD} 16^{+}$"intermediate," and CD $14^{+} \mathrm{CD} 16^{++}$ "nonclassical" monocytes (21). These subsets vary in differentiation properties, migratory capabilities, and cytokine production. The two $\mathrm{CD} 16^{+}$subsets appear to have more activated, proinflammatory phenotypes (22-24). Increased proportions of circulating CD16 ${ }^{+}$intermediate and nonclassical monocytes have been associated with acute falciparum malaria (25-27). Evidence suggests that the CD16 ${ }^{+}$subpopulations have enhanced antiparasitic activity $(26,28,29)$, though there are still significant gaps in our understanding of the functional roles of monocyte subpopulations during malaria. In this study, we characterized systemic inflammation and the monocyte subset phenotypes, monocyte functions relevant to malaria pathogenesis, and monocyte gene expression profiles of Kenyan children at the time of presentation with acute uncomplicated malaria and 6 weeks later, when they were asymptomatic following administration of antimalarial drugs. These data were compared with results of studies performed using plasma and monocytes from healthy asymptomatic infected and uninfected Kenyan children and adults living in the same community.

\section{Results}

Clinical presentations and laboratory features of the study population. The study was conducted in an area of western Kenya where transmission of $P$. falciparum and child malaria morbidity are increasing, despite the widespread distribution of long-lasting insecticidal nets $(30,31)$. Demographic features and pertinent clinical laboratory results for children with acute uncomplicated malaria, their samples obtained 6 weeks after treatment (6-week recovery samples), and healthy asymptomatic children and adults from the same communities where malaria cases were recruited are presented in Supplemental Table 1 (supplemental material available online with this article; https://doi.org/10.1172/jci.insight.95352DS1). The median ages of children with acute malaria and healthy community children were similar (4.8 and 5.7 years). The median density of peripheral blood asexual parasitemia of children with acute malaria was $48,870 / \mu 1$. Blood smears were $P$. falciparum negative for all uncomplicated malaria cases 2 weeks after a 3-day course of artemether-lumefantrine; at 6 weeks, all of these children were asymptomatic (no fever or malaria symptoms), but $41 \%$ were reinfected as detected by conventional PCR or blood smear. There was no difference in age between children with acute malaria who had asymptomatic parasitemia at their 6-week recovery visit compared with children who remained uninfected (PCR negative) at 6 weeks (median 4.8 years in both groups). The proportions of healthy community control children and adults with asymptomatic parasitemia (blood smear or PCR positive) were $52.5 \%$ and $42.8 \%$, respectively. The median plasma level of P. falciparum histidine-rich protein 2 (PfHRP2) at the time of presentation with acute malaria was $131.3 \mathrm{ng} /$ $\mathrm{ml}$, consistent with the parasite biomass associated with uncomplicated disease (32). Plasma PfHRP2 levels correlated directly with the density of asexual parasitemia (Supplemental Figure 1). Absolute leukocyte and monocyte counts among the four groups were similar. Granulocyte counts were increased and lymphocyte counts were decreased during acute malaria compared with recovery values and those of healthy children $(P<0.0001)$. The platelet count of children with acute malaria was lower than that of the other 

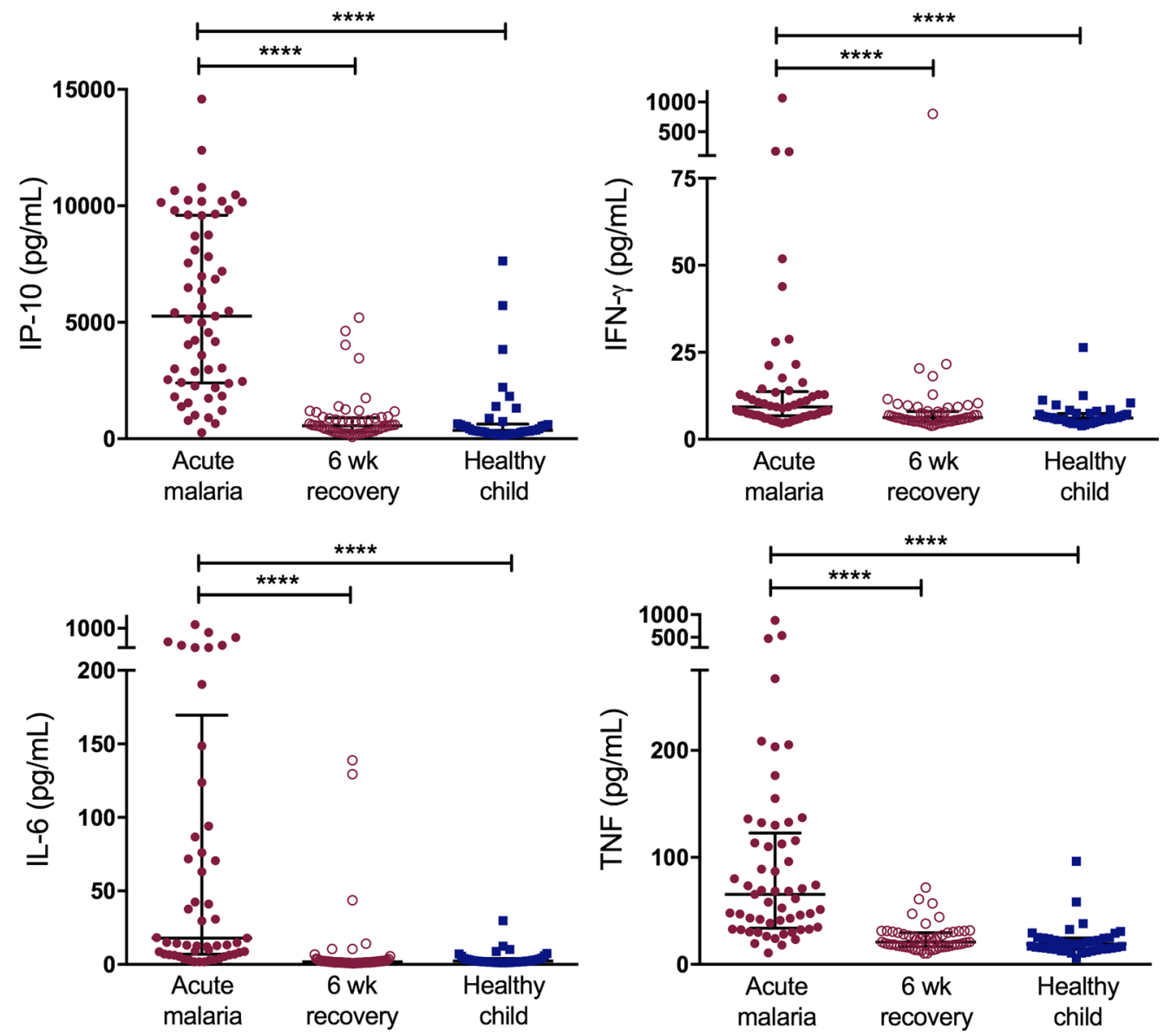

Figure 1. Acute uncomplicated malaria is associated with a robust proinflammatory cytokine response and systemic inflammation. Plasma cytokine and acute-phase reactant levels were compared in samples from cases of acute uncomplicated malaria and 6 -week recovery samples ( $n=60$ pairs) and samples from heathy child controls $(n=40)$. Bars represent medians with interquartile ranges. Wilcoxon matched-pairs rank test was used to compare acute malaria samples to 6 -week recovery samples. Kruskal-Wallis test was used to compare healthy child samples to acute malaria and 6-week recovery samples. ${ }^{* * *} P<0.0001$. IP-10, IFN- $\gamma$-induced protein 10.

groups but within the normal range for this parameter. Hemoglobin levels were lower $(P<0.05)$ at the time of presentation with acute malaria relative to recovery values at 6 weeks and those of healthy children in the same community.

Cytokines and acute-phase reactants in acute malaria and asymptomatic parasitemia. Plasma levels of 16 cytokines and acute-phase reactants were compared for acute malaria cases, their matching 6-week recovery samples, and healthy child community controls. Compared with 6-week recovery samples and samples from healthy children in the community, acute malaria was associated with a 1.5- to 9.4-fold increase in circulating levels of the proinflammatory cytokines IFN- $\gamma$-induced protein 10 (IP-10, also known as CXCL10), IFN- $\gamma$, TNF, and IL-6 (Figure 1). IL-10, which is produced by cells of both the innate and adaptive immune systems and has diverse antiinflammatory as well as antimicrobial functions, was elevated 51.2-fold during acute malaria $(P<0.0001)$. Plasma levels of soluble TNF receptors 1 and 2 (sTNF-R1 and sTNF-R2), soluble CD14, soluble B cell-activating factor (BAFF), and C-reactive protein (CRP) were also elevated during acute malaria $(P<0.0001)$, indicative of a systemic inflammatory state. Data for all 16 plasma cytokines and acute-phase reactants are presented in Supplemental Table 2. Plasma IL-10, IL-6, and TNF correlated directly with PfHRP2 levels ( $r=0.5,0.6$, and 0.7 , respectively, $P<0.001)$ during acute malaria. In contrast, plasma IFN- $\gamma$ and IL-12p70 correlated inversely with PfHRP2 levels $(r=-0.3, P<0.05)$ (Supplemental Figure 1). There was no relationship between PfHRP2 and the remaining plasma cytokines.

We also compared plasma cytokine profiles of healthy community control children with asymptomatic infection determined by positive blood smear, with asymptomatic infection determined by positive $P$. falciparum PCR (blood smear negative), and without P. falciparum infection ( $P$. falciparum PCR negative). Of note, healthy 
A Exclude doublets

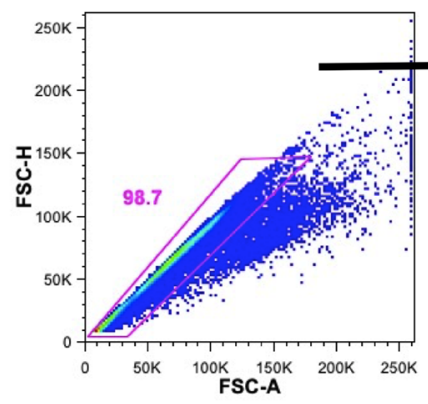

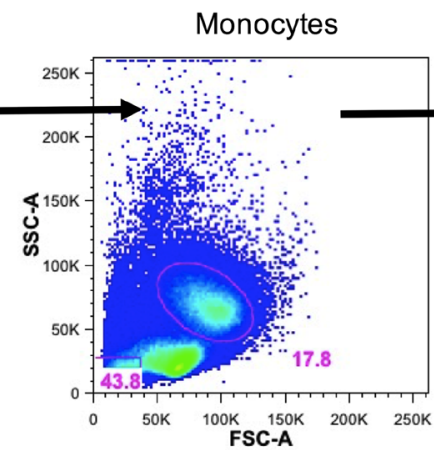

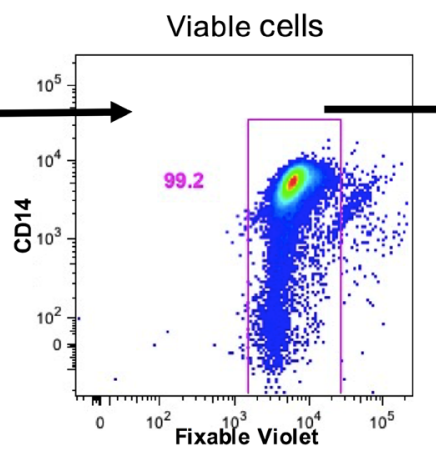

Viable cells

C

D

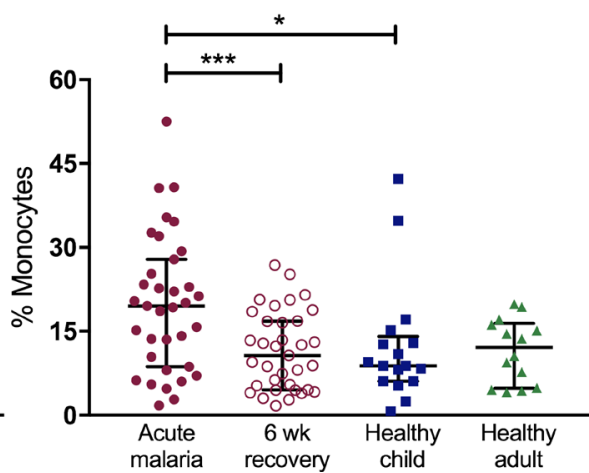

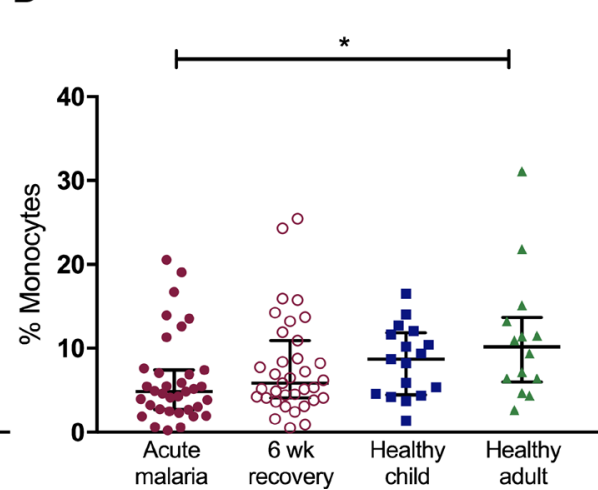

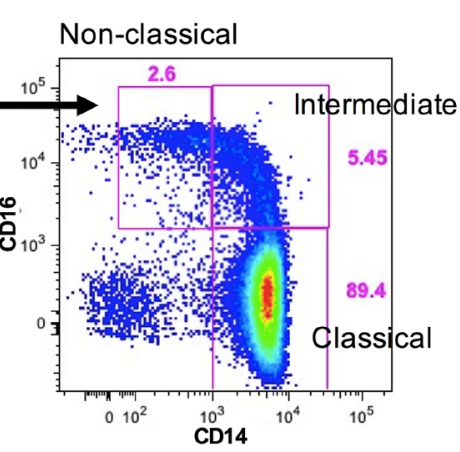

Non-classical
B

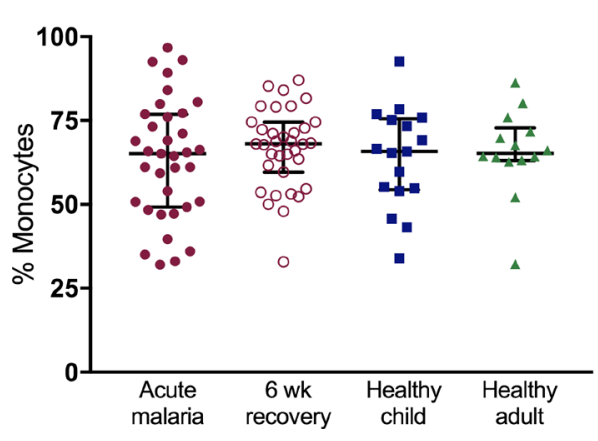

Figure 2. Acute uncomplicated malaria is associated with an expansion of the intermediate monocyte subset. (A) Gating strategy to calculate proportions of circulating monocyte subsets. Doublets were excluded, and monocytes were gated based on forward- and side-scatter properties. Dead cells were excluded based on Fixable Violet staining. The 3 subsets were determined based on CD14 and CD16 expression (classical CD14 ${ }^{++}$CD16-, intermediate $\mathrm{CD}_{14}{ }^{++} \mathrm{CD} 16^{+}$, and nonclassical $\left.\mathrm{CD} 14^{+} \mathrm{CD} 16^{++}\right)$. Proportions of all circulating monocytes that are (B) classical, (C) intermediate, and (D) nonclassical for samples from cases of acute malaria, 6-week recovery $(n=35$ pairs), healthy child controls $(n=17)$, and healthy adult controls $(n=14)$. Bars represent the median values with interquartile ranges. Wilcoxon matched-pairs rank test was used to compare acute malaria to 6 -week recovery samples. Kruskal-Wallis test was used to compare unmatched groups. ${ }^{*} P<0.05,{ }^{* *} P<0.001$.

child controls had no episodes of febrile malaria or receipt of antimalarials in the 2 months preceding study enrollment. Low-grade inflammation was associated with asymptomatic blood-stage infection detectable by peripheral blood smear, with elevated levels of TNF, IL-10, and CRP when compared with children with positive $P$. falciparum PCR but negative blood smear and uninfected children $(P<0.05)$. There was a trend toward increased plasma levels of IP-10, sTNF-R1, and sTNF-R2 in children with asymptomatic infection compared with uninfected children, though these differences were not statistically significant (Supplemental Table 3).

Monocyte subsets and surface markers of innate and adaptive immunity. Monocyte subset proportions were determined by flow cytometry. The gating strategy is shown in Figure 2A. There was no difference in the proportions of the classical $\mathrm{CD} 14^{++} \mathrm{CD} 16^{-}$monocyte subset for samples from acute malaria cases, 6-week recovery samples, and samples from healthy Kenyan child and adult controls (Figure 2B). In contrast, acute malaria was associated with an expansion of intermediate CD $14^{++} \mathrm{CD} 16^{+}$monocytes compared with 6-week recovery samples and samples from healthy child controls (median values $19.5 \%$ vs. $10.6 \%$ and $8.8 \%$, respectively; $P<0.05$; Figure $2 \mathrm{C}$ ). Healthy adults had higher proportions of nonclassical $\mathrm{CD} 14^{+} \mathrm{CD} 16^{++}$monocytes compared with children with acute malaria (median values $10.2 \%$ vs. $4.8 \% ; P$ $=0.02$ ), but there was no difference among samples from children with acute malaria, 6-week recovery samples, and samples from healthy community children (Figure 2D).

Cell surface expression of markers important to innate and adaptive immunity were altered on the three monocyte subsets during acute malaria compared with 6-week recovery samples and samples from healthy child controls. TLR2 and TLR4 expression (Figure 3A) was increased on intermediate monocytes during acute malaria compared with recovery samples and samples from healthy children, with no differences in expression on the classical or nonclassical subsets. In contrast, surface expression of the scavenger receptor CD36, which is important to nonopsonic phagocytosis of IEs, was decreased on both classical and nonclassical subsets during 
A

TLR2

TLR4

CD36
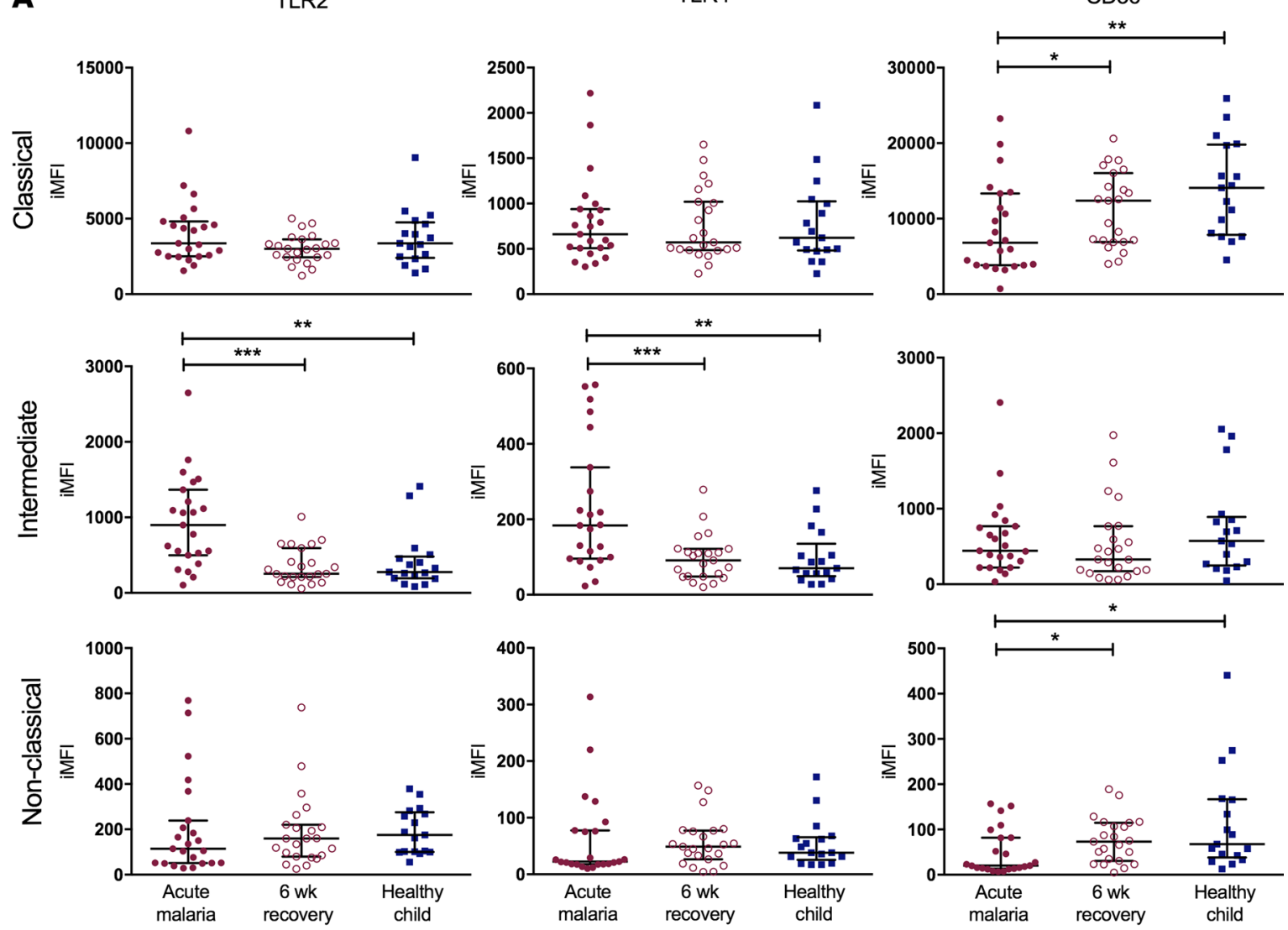

B

BAFF

PD-L1
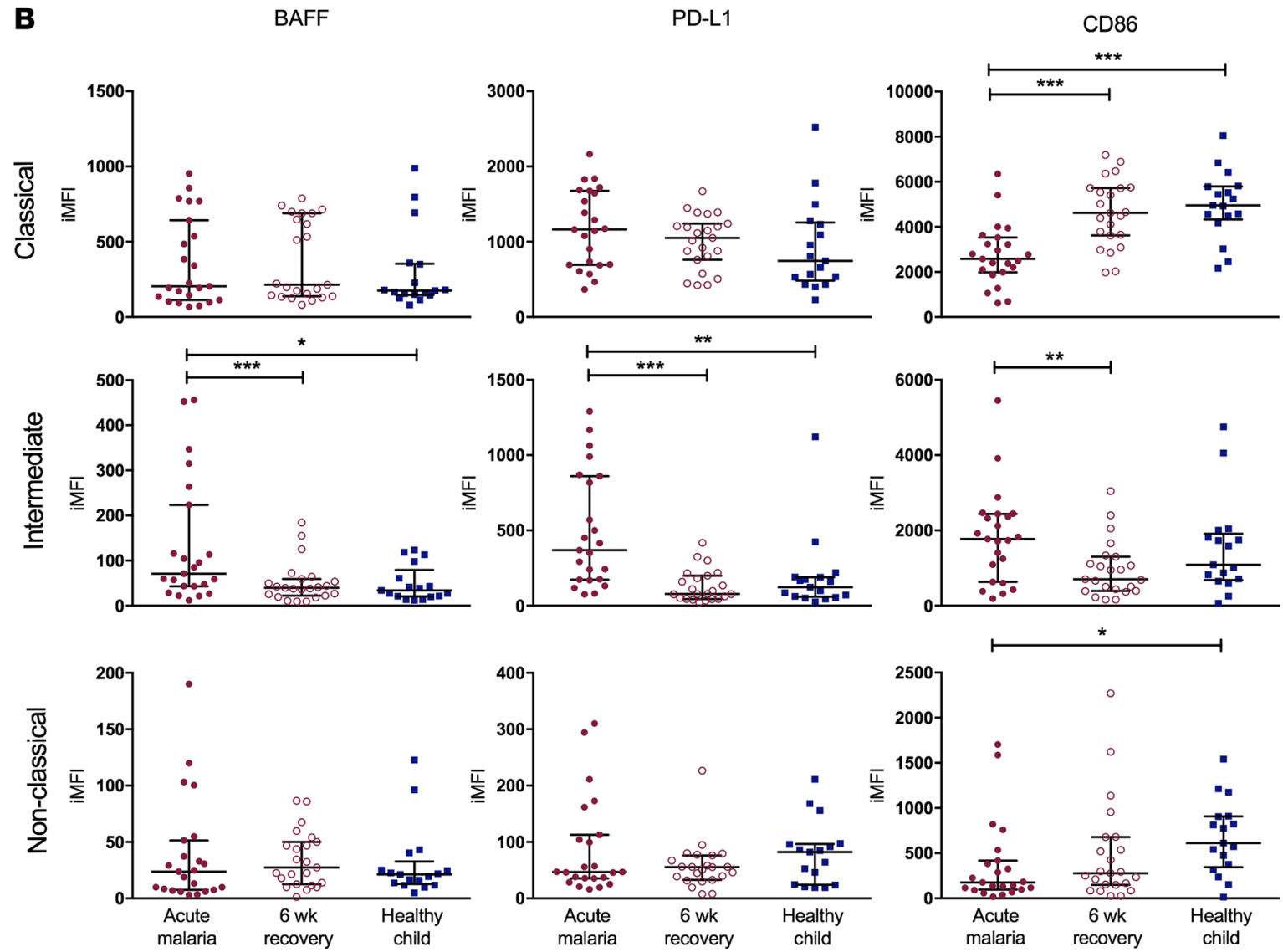
Figure 3. Cell surface phenotypes of monocyte subsets are activated during acute uncomplicated malaria. Flow cytometry was used to determine cell surface expression of 6 markers on the three monocyte subsets (classical, intermediate, and nonclassical) for samples from cases of acute malaria, 6-week recovery samples ( $n=23$ pairs), and samples from healthy child controls $(n=17)$. (A) Cell surface expression of markers important in innate immunity: TLR2, TLR4, and CD36. (B) Cell surface expression of markers important in adaptive immunity: BAFF, PD-L1, and CD86. Wilcoxon matched-pairs rank test was used to compare acute malaria samples to 6-week recovery samples. Kruskal-Wallis test was used to compare healthy child samples to acute malaria and 6-week recovery samples. Bars represent the median values with interquartile ranges. ${ }^{*} P<0.05,{ }^{* *} P<0.01,{ }^{* *} P<0.001,{ }^{* * * *} P<0.0001$. iMFI, integrated mean fluorescence intensity; BAFF, B cell-activating factor; PD-L1, programmed death-ligand 1.

acute disease. With respect to adaptive immunity (Figure 3B), surface expression of BAFF, a critical regulator of B cell maturation and survival, was increased on intermediate monocytes during acute malaria. Programmed death-ligand 1 (PD-L1), which delivers inhibitory signals upon binding to its receptor on $\mathrm{T}$ cells, had increased expression on intermediate monocytes during acute malaria. CD86 delivers costimulatory signals needed for T cell activation, and while CD86 expression was increased on intermediate monocytes during acute disease, its expression was significantly decreased on both the classical and nonclassical subsets. Increased ratios of PD-L1/ CD86 expression on intermediate monocytes have been described in individuals with chronic hepatitis $\mathrm{C}$ infection and were associated with increased viremia and impaired antiviral functions (33). The ratio of PD-L1/ CD86 expression was increased on all monocyte subsets during acute malaria compared with 6-week recovery samples and samples from healthy children (Figure 4).

We also compared monocyte subset proportions and innate and adaptive immunity phenotypic markers of healthy control children with asymptomatic parasitemia versus those who were asymptomatic with no $P$. falciparum infection detectable by blood smear or PCR. While monocyte subset proportions were similar between children with asymptomatic infection and those who were uninfected (Supplemental Figure 2), children with asymptomatic infection had altered expression of TLR4, CD36, BAFF, and CD86 on monocyte subsets compared with uninfected children (Supplemental Figure 3).

Monocyte phagocytic function during symptomatic and asymptomatic infection. We assessed both nonopsonic and opsonic phagocytic activity of the participants' monocytes during acute malaria compared with that 6 weeks following treatment. Peripheral blood mononuclear cells (PBMCs) were incubated with plasma-opsonized CFSE-labeled IEs, and phagocytosis by monocytes was quantified by flow cytometry. When IEs were opsonized with heat-inactivated North American plasma, monocyte phagocytic activity was presumed to comprise primarily nonopsonic phagocytosis, as complement is inactivated by heat and malaria-naive North American plasma does not contain IE-specific antibodies. When IEs were opsonized with heat-inactivated pooled plasma from malaria-immune Kenyan adults, monocyte phagocytic activity was presumed to represent primarily IE-specific IgG-mediated opsonic phagocytosis. Adults living in this area of western Kenya have high levels of antibodies specific for P. falciparum variant surface antigens (VSAs) expressed on the surface of IEs, including VSAs expressed by the Ghana14 isolate used in phagocytosis assays (Supplemental Figure 4A). Because phagocytosis assays show day-to-day experimental variation (generally due to small variations in magnet purification of IEs), all assays were performed in parallel with North American malaria-naive PBMCs (from a single donor) run alongside each acute-recovery PBMC pair (Supplemental Figure 4, B and C). Each acute-recovery pair was run in the same-day assay, so comparisons were made of phagocytic function between acute-recovery pairs but not of absolute differences in phagocytosis among individuals.

Opsonic phagocytic activity of all monocytes was decreased during acute malaria compared with that 6 weeks after treatment (median values $29.8 \%$ phagocytosis vs. $43.8 \%, P=0.02, n=12$ pairs; Figure 5A). These results appear to be driven by 6 acute-recovery pairs of children who were uninfected ( $P$. falciparum PCR negative) at recovery (23.6\% phagocytosis during acute malaria vs. $40.2 \%$ upon recovery, $P=0.03$; Figure $5 \mathrm{~B})$, as there was no difference in monocyte opsonic phagocytic function between acute malaria and recovery samples from the 6 children who had asymptomatic infection at recovery ( $40.8 \%$ vs. $46.1 \%$ respectively, $P=$ 0.56 ; Figure $5 \mathrm{C}$ ). There was no statistically significant difference in nonopsonic phagocytosis between acute malaria and recovery samples from all 12 children ( $4.7 \%$ vs. $6.2 \%, P=0.24$; Figure 5D). A small decrease in nonopsonic phagocytic function during acute malaria compared with recovery was also noted in acute-recovery pairs of children who were uninfected at recovery ( $4.5 \%$ vs. $7.2 \%, P=0.03$; Figure $5 \mathrm{E})$. As with opsonic phagocytic function, there was no difference in monocyte nonopsonic phagocytic function between acute malaria and recovery in children who had asymptomatic infection at recovery $(6.2 \%$ vs. $5.6 \%, P=0.44$; Figure $5 \mathrm{~F}$ ). Taken together, these data show that $P$. falciparum infection causing uncomplicated malaria disease as well as asymptomatic parasitemia is associated with impaired monocyte phagocytosis of IEs. 
A

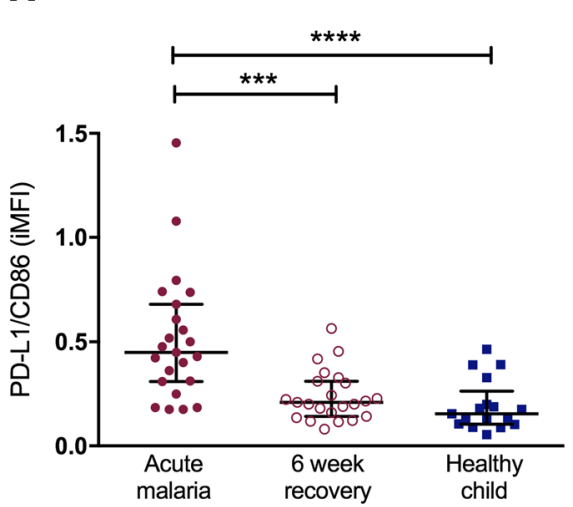

B

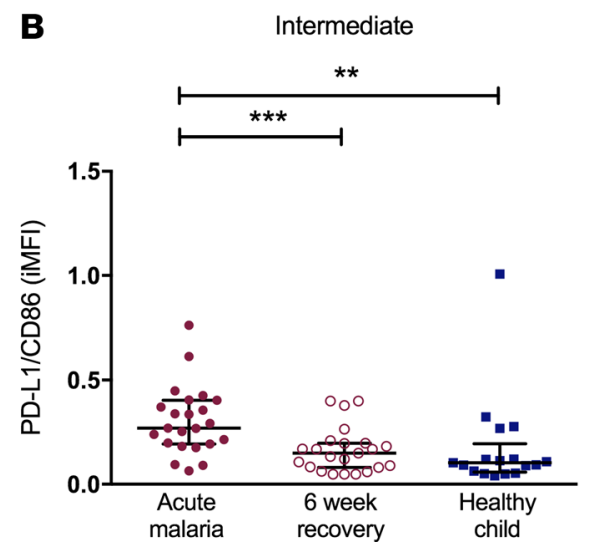

C

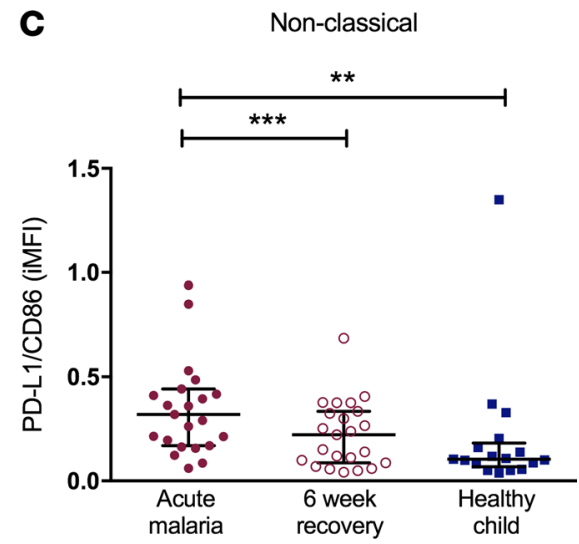

Figure 4. Acute uncomplicated malaria is associated with an increased PD-L1/CD86 ratio on all monocyte subsets. Ratios of cell surface expression of PD-L1/CD86 on the (A) classical, (B) intermediate, and (C) nonclassical monocyte subsets for samples from cases of acute malaria, 6-week recovery samples ( $n=23$ pairs), and samples from healthy child controls $(n=17)$ are indicated on the $y$ axes. Bars represent the median values with interquartile ranges. Wilcoxon matched-pairs rank test was used to compare acute malaria samples to 6-week recovery samples. Kruskal-Wallis test was used to compare healthy children to acute malaria samples and 6-week recovery samples. ${ }^{* *} P<0.01,{ }^{* * *} P<0.001$, ${ }^{* * *} P<0.0001$. iMFI, integrated mean fluorescence intensity; PD-L1, programmed death-ligand 1.

IE phagocytosis by monocyte subsets. Differences in phagocytic function were analyzed across the three monocyte subsets within a given individual at the time of presentation with and recovery from acute malaria. During acute malaria, both intermediate and nonclassical monocytes displayed greater opsonic phagocytic activity compared with classical monocytes $(48.1 \%$ and $53.6 \%$ vs. $26.4 \%, P<0.01$; Figure 6 A). Similar patterns were seen for monocytes from 6-week recovery samples: nonclassical monocytes displayed greater opsonic phagocytic activity compared with classical monocytes ( $66 \%$ vs. $34.6 \%, P=0.003$; Figure $6 \mathrm{~A}$ ).

Nonopsonic phagocytic activity was also greater in the intermediate and nonclassical subsets. During acute malaria, intermediate and nonclassical monocytes displayed greater nonopsonic phagocytic activity compared with classical monocytes $(7.6 \%$ and $6.7 \%$ vs. $4.5 \%, P<0.05$; Figure $6 \mathrm{~B})$. Similar patterns were seen 6 weeks following treatment, in which intermediate and nonclassical monocytes had greater nonopsonic phagocytic function compared with classical monocytes ( $14 \%$ and $10.3 \%$ vs. $4.9 \%, P<0.05$; Figure $6 \mathrm{~B}$ ). These patterns of differential phagocytic function among the monocyte subsets were similar in children who were uninfected at recovery and those with asymptomatic parasitemia at recovery (Figure 6, C-F).

Monocyte cytokine responsiveness to TLR ligands during acute malaria and upon recovery. Monocyte cytokine production was analyzed using negatively selected monocytes isolated from fresh venous blood samples from children presenting with acute malaria and 6 weeks following treatment. Freshly isolated monocytes from malaria-naive North American adults were used as experimental controls. Isolated monocytes were cultured for 18 hours, and constitutive cytokine production (media alone) was compared with stimulation with a TLR4 agonist (LPS) and a TLR2/TLR1 agonist (Pam3CSK4 [P3C]). Monocytes from both acute malaria and recovery visits were highly responsive to stimulation with LPS and P3C, without signs of innate immune tolerance. For example, monocytes from acute malaria cases showed an over 300-fold increase in IL-6 production and an over 100-fold increase in TNF production after stimulation with LPS. A similar effect was seen for 6-week recovery monocytes, with a 90-fold increase in IL-6 and an over 100-fold increase in TNF after LPS stimulation (Figure 7). There were no statistically significant differences in cytokine responses to LPS or P3C between acute and recovery monocytes (the only statistically significant difference between acute malaria and recovery monocytes was in constitutive production of IL-12p40) (Figure 7). Half of these children had asymptomatic parasitemia at their 6-week recovery visit. TLR responses were not different between those who remained uninfected ( $P$. falciparum PCR negative) at 6 weeks and those with asymptomatic parasitemia (blood smear or PCR positive) (Supplemental Figure 5).

Monocytes from healthy malaria-naive North Americans were included as experimental controls (representing nontolerant monocytes) to ensure that the assay worked. In this regard, it is notable that monocytes from Kenyan children during acute malaria and upon recovery showed greater magnitude of proinflammatory cytokine production in response to stimulation with TLR ligands than did healthy North American monocytes, particularly for IL-6, IL-12p40, and TNF (Figure 7). Collectively, these data show no evidence for 


\section{All monocytes Opsonic}

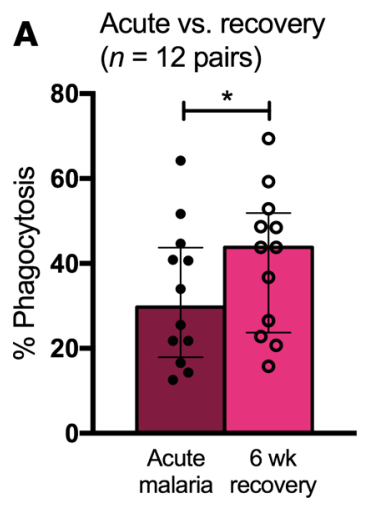

B Acute vs. Pf PCR negative recovery ( $n=6$ pairs)

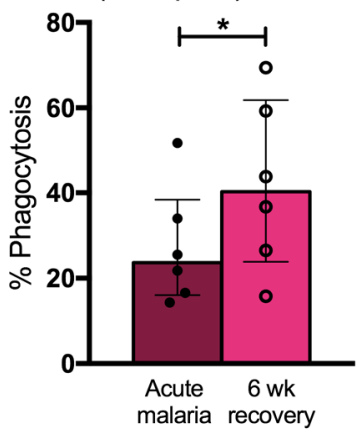

C Acute vs. Pf PCR or blood smear positive recovery

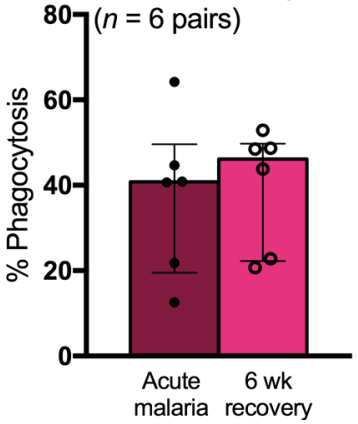

\section{All monocytes Non-opsonic}

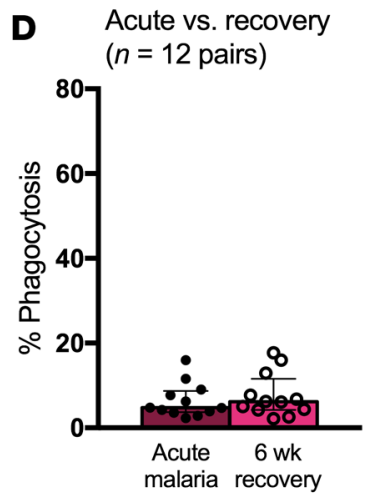

E

Acute vs. Pf PCR negative recovery ( $n=6$ pairs)

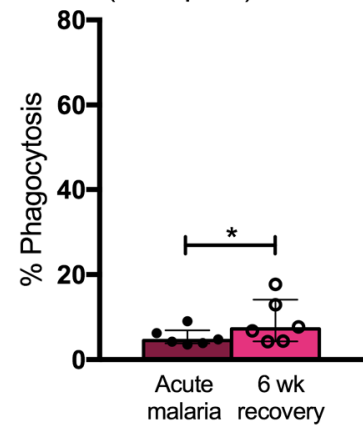

$\mathbf{F}$

\section{F Acute vs. Pf PCR or blood smear} positive recovery

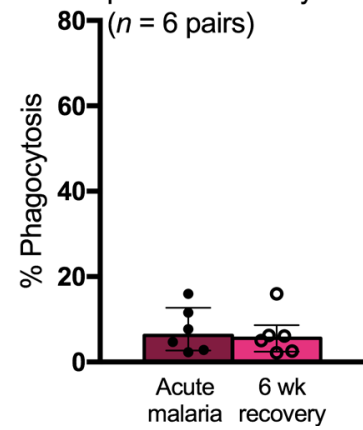

Figure 5. Monocytes display decreased phagocytic function during $P$. falciparum infection. (A) Opsonic phagocytic function of all monocytes from acute malaria (AM) and 6-week recovery PBMC samples ( $n=12$ pairs) in the presence of Ghana14 IEs opsonized with heat-inactivated pooled plasma from Kenyan adults. (B) Opsonic phagocytic function of all monocytes from AM and 6-week PBMC samples ( $n=6$ pairs) from a subset of children who were $P$. falciparum PCR negative at 6-week follow up. (C) Opsonic phagocytic function of all monocytes from AM and 6-week PBMC samples ( $n=6$ pairs) from a subset of children with asymptomatic $P$. falciparum infection at 6 -week follow up (defined by positive blood smear or $P$. falciparum PCR). (D) Nonopsonic phagocytic function of all monocytes from AM and 6-week PBMC samples $(n=12$ pairs) in the presence of Ghana14 IEs opsonized with heat-inactivated plasma from a malaria-naive North American. (E) Nonopsonic phagocytic function of all monocytes from AM and 6 -week PBMC samples ( $n=6$ pairs) from a subset of children who were $P$. falciparum PCR negative at 6-week follow up. (F) Nonopsonic phagocytic function of all monocytes from AM and 6-week PBMC samples ( $n=6$ pairs) from a subset of children with asymptomatic $P$. falciparum infection at 6 -week follow up (defined by positive blood smear or $P$. falciparum $\mathrm{PCR}$ ). Wilcoxon matched-pairs rank test was used to compare AM to 6 -week recovery samples. Data are shown as medians with interquartile ranges. ${ }^{*} P<0.05$.

monocyte tolerance during or after recovery from acute malaria and, in fact, suggest that monocytes from these children are primed to respond to TLR4 and TLR2/TLR1 ligands independent of $P$. falciparum infection status.

Monocyte gene expression profiles during acute malaria and upon recovery. Using a targeted panel for digital RNA sequencing, we characterized inflammatory gene expression profiles of monocytes isolated from six children during acute malaria and their matching samples 6 weeks following treatment. All of these children were P. falciparum PCR negative at 6 weeks. Targeted analysis of 508 genes involved in inflammation and immunity identified 125 differentially expressed genes (51 overexpressed and 74 underexpressed; $P<0.05$ ) during malaria compared with monocytes obtained 6 weeks after treatment. The heatmap in Figure 8A illustrates key genes that were differentially expressed during acute malaria compared with recovery. Monocyte genes overexpressed during acute malaria included several important inflammatory genes, including complement proteins and genes involved in TLR signaling (TLR8, LY96, MYD88). Expression of Epstein-Barr virus induced 3 (EBI3) was increased approximately 30-fold $(P<0.0001)$ during acute malaria relative to recovery. $E B I 3$ encodes the IL-27 $\beta$ subunit of the cytokines IL-27, which has both proinflammatory and antiinflammatory roles in regulating cell-mediated immunity (34), and IL-35, an antiinflammatory cytokine important in Treg function $(35,36)$. Notably, monocytes during acute malaria also underexpressed several genes involved in the inflammatory response and chemotaxis, including numerous chemokines and chemokine receptors, genes important to T cell activation (CD86, CIITA, CTLA4), and transcriptional regulators of the inflammatory response (STAT4, REL, HIF1A). These data indicate that, by the time these patients present to the clinic with fever and other symptoms of uncomplicated malaria, their monocytes may have already started to downregulate an initial proinflammatory response.

Pathway enrichment analysis of the genes overexpressed during acute malaria compared with recovery revealed significant enrichment in two Kyoto Encyclopedia of Genes and Genomes pathways: "Fc gamma receptor mediated phagocytosis" ( $P=0.0007,7$ genes) and "Staphylococcus aureus infection" ( $P=0.002,9$ genes) (Supplemental Table 4). Interestingly, genes for several proinflammatory mediators (including IL-1 $\beta$, IL-6, IL-8, and TNF) were not differentially expressed between acute malaria and recovery, even though plasma cytokine profiles revealed a robust proinflammatory cytokine response during acute disease. The heatmap in Figure $8 \mathrm{~B}$ illustrates several important inflammatory genes that were not differentially expressed by monocytes between acute and recovery but were significantly different from monocytes from healthy North American malaria-naive controls 
Phagocytic function of monocyte subsets Opsonic
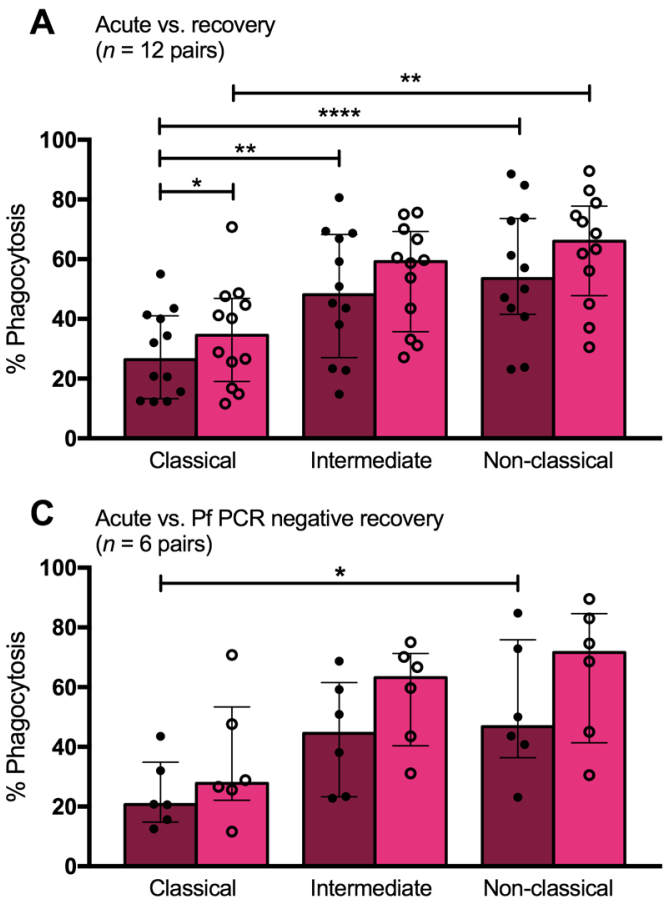

E Acute vs. Pf PCR or blood smear positive recovery ( $n=6$ pairs)

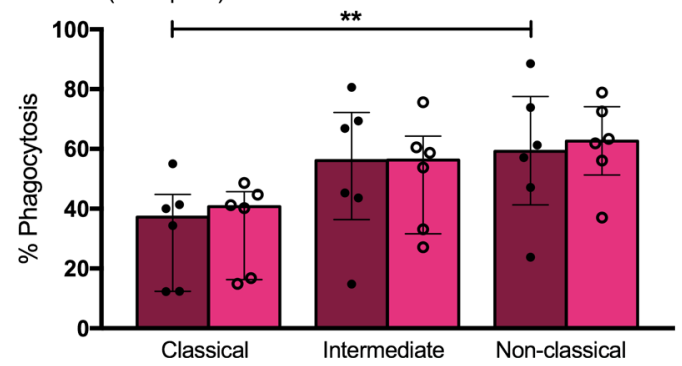

Phagocytic function of monocyte subsets Non-opsonic

B Acute vs. recovery ( $n=12$ pairs)
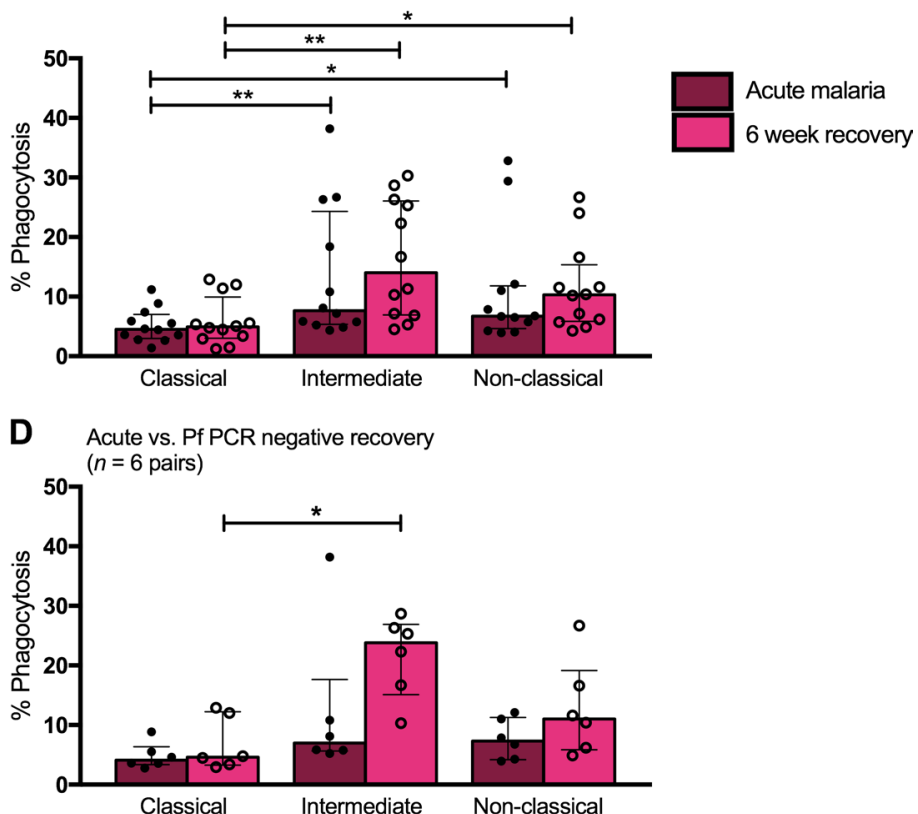

F Acute vs. Pf PCR or blood smear positive recovery ( $n=6$ pairs)

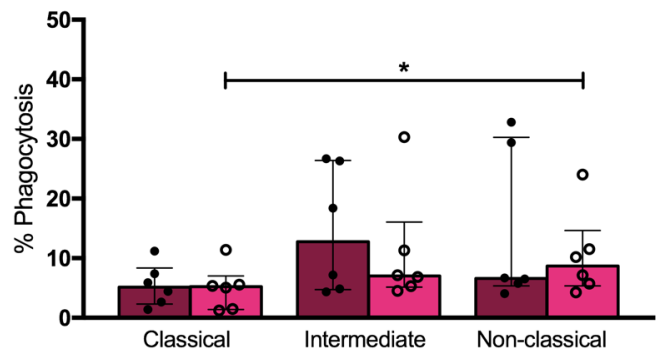

Figure 6. Intermediate and nonclassical monocytes display greater phagocytic function compared with classical monocytes. (A) Opsonic phagocytic function of the three monocyte subsets from acute malaria (AM) and 6-week recovery PBMC samples $(n=12$ pairs) in the presence of Ghana14 IEs opsonized with heat-inactivated pooled plasma from malaria-immune Kenyan adults. (B) Nonopsonic phagocytic function of monocyte subsets from AM and 6-week PBMC samples ( $n=12$ pairs) in the presence of Ghana14 IEs opsonized with heat-inactivated plasma from a malaria-naive North American. (C) Opsonic phagocytic function of monocyte subsets from AM and 6-week PBMC samples ( $n=6$ pairs) from a subset of children who were $P$. falciparum PCR negative at 6 weeks. (D) Nonopsonic phagocytic function of monocyte subsets from AM and 6-week PBMC samples ( $n=6$ pairs) from a subset of children who were $P$. falciparum PCR negative at 6 weeks. (E) Opsonic phagocytic function of monocyte subsets from AM and 6 -week PBMC samples ( $n=6$ pairs) from a subset of children with asymptomatic $P$. falciparum infection at 6 weeks (defined by positive blood smear or $P$. falciparum PCR). (F) Nonopsonic phagocytic function of monocyte subsets from AM and 6-week PBMC samples ( $n=6$ pairs) from a subset of children with asymptomatic $P$. falciparum infection at 6 weeks (defined by positive blood smear or $P$. falciparum PCR). Differences between AM and 6-week PBMC samples analyzed by Wilcoxon matched-pairs rank test. Differences among subsets analyzed by Friedman test with multiple comparisons. Data shown are medians with interquartile ranges. ${ }^{*} P<0.05,{ }^{* *} P<0.01,{ }^{* * *} P<0.0001$.

$(P<0.05)$. Of the 508 genes analyzed, 114 were overexpressed and 86 underexpressed during acute malaria compared with North American controls; 124 were overexpressed and 95 underexpressed at the 6-week recovery visit compared with North American controls $(P<0.05)$. There was substantial overlap for these differentially expressed genes, as 75 genes were overexpressed for both acute malaria and 6-week recovery monocytes compared with North American controls. These gene expression data suggest that monocytes from these children (during acute malaria and in a healthy state) are tightly regulated and primed for an inflammatory response. This is consistent with our results showing that monocytes are equally responsive to TLR stimulation during acute malaria as upon recovery 

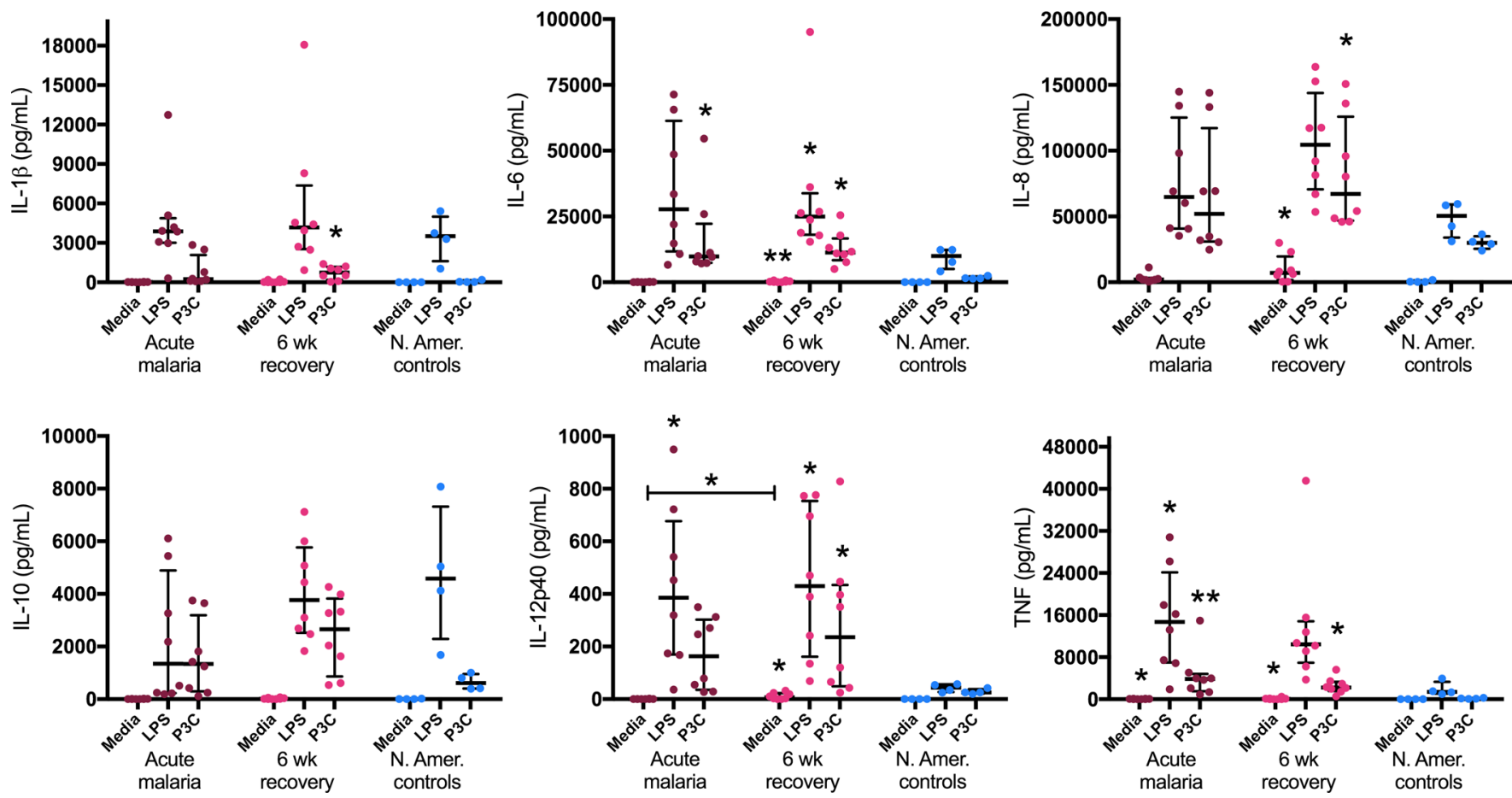

Figure 7. Monocytes from children during acute malaria and 6 weeks following treatment are highly responsive to stimulation with TLR ligands. Monocytes were negatively selected from fresh venous blood samples from children during acute malaria and 6 weeks following treatment ( $n=8$ pairs). Healthy North American (N. Amer.) adult controls $(n=4)$ were used as experimental controls. All assays were performed with technical duplicates. Cells were cultured for 18 hours with media alone, $10 \mathrm{ng} / \mathrm{ml}$ LPS, or $100 \mathrm{ng} / \mathrm{ml}$ Pam3CSK4 (P3C), and cytokine concentrations were measured in culture supernatants. Wilcoxon matched-pairs rank test was used to compare acute malaria to 6 -week recovery samples. Kruskal-Wallis test was used to compare acute malaria, 6-week recovery, and N. Amer. control samples. Data are shown as medians with interquartile ranges. ${ }^{*} P<0.05,{ }^{*} P<0.01$, as compared with controls.

(with enhanced TLR responses relative to malaria-naive monocyte controls). Of note, gene expression profiles of individual monocyte subsets were not analyzed separately due to the limited number of PBMCs obtained from these pediatric patients.

\section{Discussion}

We demonstrate in this report significant changes in monocyte phenotypes and functions associated with symptomatic and asymptomatic $P$. falciparum infection of Kenyan children living in a malaria endemic area. During uncomplicated malaria, an expansion of the inflammatory intermediate $\mathrm{CD} 14^{++} \mathrm{CD} 16^{+}$ monocyte subset was coupled with acutely activated monocyte phenotypes. Impairment in monocyte phagocytic function was associated with both symptomatic and asymptomatic $P$. falciparum infection. Freshly isolated monocytes from both acute and recovery time points demonstrated primed TLR responses. Monocyte transcriptional profiles reflected a tightly regulated response during uncomplicated malaria compared with recovery, with balanced proinflammatory and antiinflammatory gene expression. In addition, several inflammatory genes that were expressed equally by acute and recovery monocytes had significantly increased expression when compared with healthy malaria-naive controls, consistent with our functional data showing primed TLR responses at both acute and recovery time points.

Innate immune activation plays a crucial role in host protection as well as pathogenesis during malaria infection. Dynamic shifts in monocyte subpopulations occur during symptomatic malaria, and each monocyte subset is characterized by different stages of differentiation with distinct gene expression profiles, phenotypes, and physiologic functions $(22,37)$. Our results are similar to those in which Ogonda et al. found an expansion in the intermediate $\mathrm{CD} 14^{++} \mathrm{CD} 16^{+}$subset in cases of both uncomplicated and severe malaria in children from western Kenya (27). The dynamics of circulating human monocyte subsets were recently studied using in vivo deuterium labeling, and experimental endotoxemia led to a transient but profound monocytopenia with restoration of circulating monocytes through release of classical monocytes from the bone marrow; classical monocytes were shown to give rise to intermediate and nonclassical 


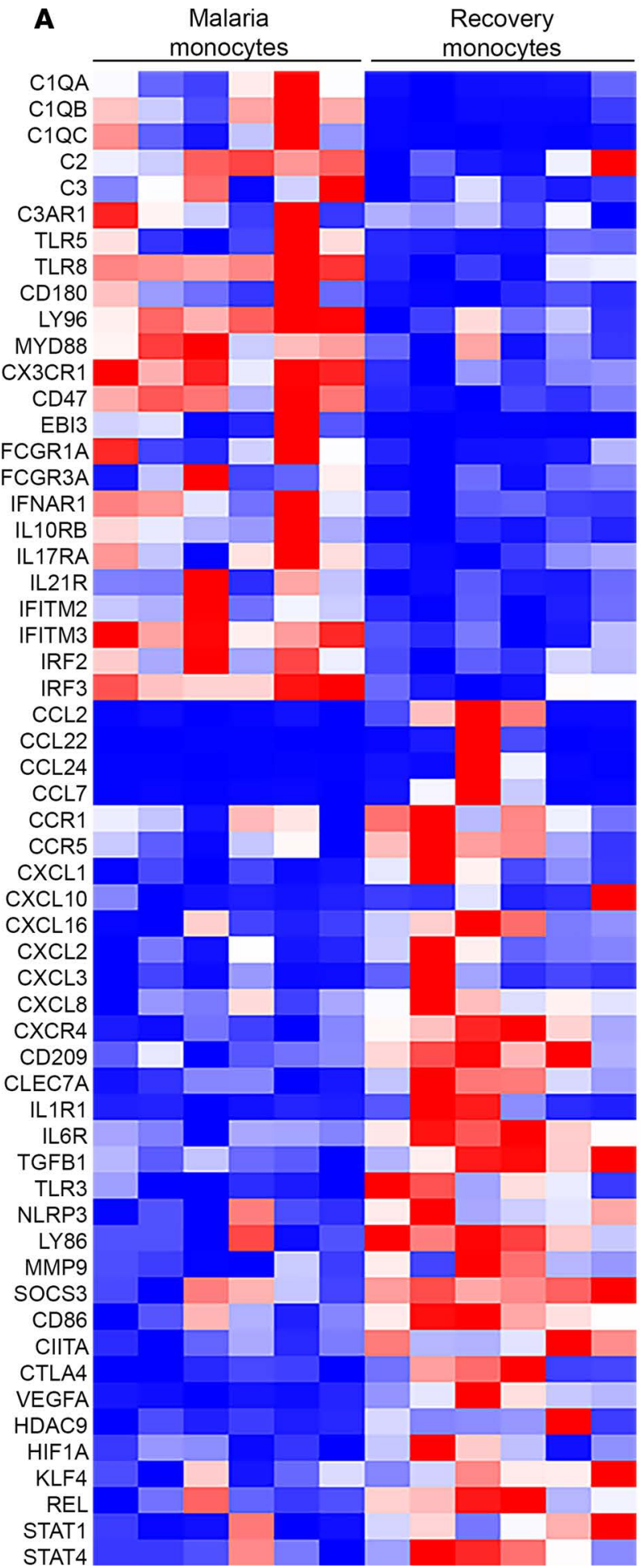

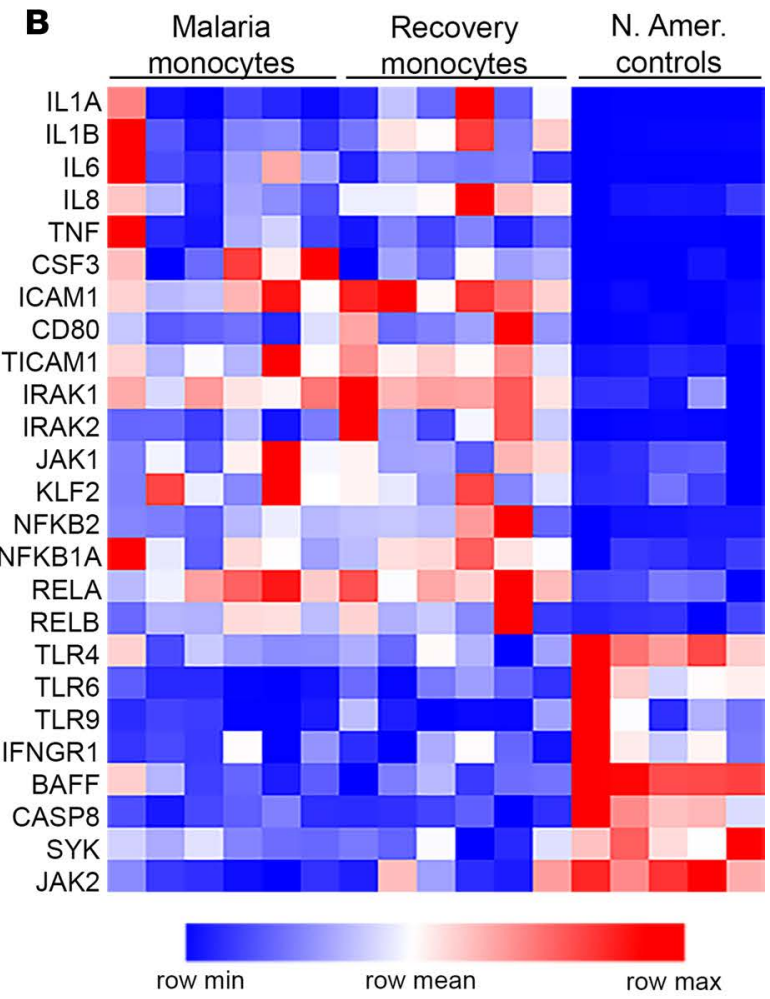

Figure 8. Monocytes from children with uncomplicated malaria have a distinct, regulated inflammatory gene expression profile. Targeted digital RNA sequencing was performed on monocytes isolated from cryopreserved PBMCs via negative selection over a magnetic column. A customized panel targeted 508 genes important in inflammation and immunity (QIAseq Human Inflammation and Immunity Transcriptome Panel, Qiagen). Differential expression was determined using the DESeq2 R/Bioconductor package. (A) Monocyte transcriptional profiles were compared between samples from cases of acute malaria and matching 6-week recovery samples ( $n=6$ pairs). Of the 508 genes analyzed, 125 were differentially expressed between $A M$ and 6-week recovery (51 overexpressed and 74 underexpressed during AM; $P<0.05$ ). Key genes are listed in the heatmap. (B) Monocyte transcriptional profiles were compared among samples from children with acute malaria, 6 -week recovery samples ( $n=6$ pairs), and samples from healthy malaria-naive North American controls $(n=5)$. Of the 508 genes analyzed, 114 were overexpressed and 86 underexpressed during acute malaria compared with North American controls and 124 were overexpressed and 95 underexpressed at 6-week recovery compared with North American (N. Amer.) controls $(P<0.05)$. Key genes are listed in the heatmap. None of the genes listed in $\mathbf{B}$ were differentially expressed between acute malaria and recovery.

monocytes in the periphery (38). During malaria, systemic inflammatory factors as well as local factors in the bone marrow may provide signals that lead to an increased percentage of classical monocytes that transition into intermediate (and ultimately nonclassical) monocytes (39). Increased proportions of inflammatory monocyte subpopulations may be beneficial to the host via enhanced antiparasitic functions. Strong antibody-dependent cellular inhibition activity was noted in a distinct CD16 ${ }^{+}$subpopulation of monocytes in Thai adults with falciparum malaria (26). In our study, CD16 intermediate and nonclassical monocytes had greater phagocytosis of IEs compared with the classical subset. Similarly, Antonelli et al. have shown superior phagocytosis of Plasmodium vivax-infected reticulocytes by the intermediate subset (28). 
Here, we showed that uncomplicated malaria in children was associated with monocyte activation in the context of significant systemic inflammation. Plasma levels of IL-10 and TNF were markedly elevated, and IL-10, TNF, and IL-6 positively correlated with PfHRP2 levels, while IL-12p70 and IFN- $\gamma$ negatively correlated with PfHRP2 levels. Luty et al. found remarkably similar correlations with parasitemia and plasma IL-10, TNF, and IL-12p70 levels among Gabonese children with uncomplicated and severe malaria (40). Weinberg et al. also demonstrated similarly elevated plasma levels of IL-10 in Tanzanian children with moderately severe and severe malaria, with IL-10 levels showing a significant correlation with disease severity as well as monocyte polarization toward an M2 phenotype with high levels of arginase 1 and low levels of NOS2 expression (which may contribute to NO insufficiency and oxidative stress) (41). Collectively, these observations suggest that oxidative stress related to heme and/or hemozoin toxicity to phagocytes may enhance IL-10 production while downregulating IL-12 activity (42).

We also demonstrated that asymptomatic parasitemia was associated with impaired monocyte phagocytosis, altered monocyte surface phenotypes, and elevated levels of inflammatory cytokines and acute-phase reactants. These systemic inflammatory factors are likely produced by several different cell types, including monocytes, neutrophils, and dendritic cells. The level of inflammation appears to be dependent on the density of blood-stage infection and, by inference, $P$. falciparum biomass, as children with asymptomatic infection detectable by blood smear had higher levels of inflammation compared with uninfected children as well as children who were $P$. falciparum PCR positive but blood smear negative. Others have shown an association of asymptomatic $P$. falciparum infection in children with increased proportions of circulating CD $16^{+}$ monocytes (43), anemia, and increased markers of systemic inflammation (44). Impaired phagocytosis by dendritic cells has been associated with submicroscopic parasitemia in malaria-naive adult volunteers experimentally challenged with $P$. falciparum-IEs (45). These data lend support to the assessment that the term "asymptomatic parasitemia" is inaccurate and should instead be referred to as "chronic malaria infection," given the detrimental effects that even low-density infections may have on innate immune functions and individual health and development (in addition to serious societal and public health concerns) $(46,47)$.

Impairment in monocyte phagocytosis was observed across the clinical spectrum of $P$. falciparum infection in this cohort, and further research is required to elucidate the underlying mechanisms. Previous monocyte phagocytosis of IEs in vivo is likely to impair the phagocytic capacity of these cells ex vivo. Schwarzer et al. have shown that ingestion of hemozoin impairs the ability of adherent monocytes to repeat phagocytosis (48). Plasmodium parasites might also employ mechanisms to directly manipulate the host cytoskeleton and evade phagocytosis (49). Intravascular hemolysis during malaria infection leads to release of the heme moiety from extracellular hemoglobin, and we speculate that heme toxicity contributes to impaired monocyte phagocytosis of IEs. This notion is supported by recent work by Martins et al., in which they demonstrate that heme inhibits phagocyte function directly by disrupting the actin cytoskeleton via interactions with DOCK8 and Cdc42 (50). In addition, increasingly impaired neutrophil phagocytosis of Salmonella was associated with increasingly severe hemolysis during malaria in Gambian children (51).

Another possible mechanism for impaired phagocytosis involves increased signaling through the inhibitory CD47 pathway. Recently, Ayi et al. showed that CD47-SIRP $\alpha$ interactions negatively regulate macrophage phagocytosis of IEs, and CD47 deficiency was associated with improved survival in a murine model of cerebral malaria (52). Our targeted transcriptional analysis revealed increased expression of $C D 47$ as well as enrichment in the "Fc gamma receptor mediated phagocytosis" pathway during acute malaria compared with recovery. Several genes in the Fc $\gamma$ R-mediated phagocytosis pathway were overexpressed during acute malaria, including CDC42 and genes for the activating Fc $\gamma$ Rs (FCGR1A, FCGR3A). A limitation of our targeted analysis is that expression of some key inhibitory genes in this pathway were not measured (including the inhibitory $\mathrm{F} c \gamma \mathrm{R}, F C G R 2 B$, and the downstream phosphatase $S H I P$, responsible for negative regulation of phagocytosis, ref. 53). We hypothesize that, as part of the regulated inflammatory response that occurs during uncomplicated malaria, inhibitory receptors are upregulated to control inflammation, and as a result, lead to decreased phagocytic capacity.

Monocyte activation in response to an infectious insult is generally described as a polarization toward either a tolerant or a primed phenotype. Monocyte/macrophage tolerance is well described in patients with bacterial sepsis $(54,55)$ and in animal and in vitro models of LPS hyporesponsiveness $(56,57)$. Endotoxin tolerance was initially defined as a transient unresponsive state in which cells previously exposed to endotoxin are unable to respond to repeat challenge with endotoxin (primarily measured by a decreased ability to produce proinflammatory cytokines) (58). Monocyte tolerance is now more accurately and comprehensively 
described as a reprogramming or modulation of cellular responses, rather than as global suppression or exhaustion (59). Several studies have shown that although tolerant human monocytes have reduced production of TNF and IL-6 after LPS rechallenge, along with impaired antigen presentation, these cells have enhanced or preserved antimicrobial functions, including phagocytosis, reactive oxygen species production, and tissue remodeling (60-62). Transcriptome studies in monocytes/macrophages have revealed distinct genetic signatures associated with endotoxin tolerance $(57,60)$. Shalova et al. studied transcriptome profiles of monocytes from adults with Gram-negative urosepsis and showed an overexpression of a large number of proinflammatory genes when compared with monocytes obtained after clinical recovery, including several chemokines (CCL2, CCL7), proinflammatory cytokines (IL1B, ILO), and transcription factors (HIF1A, STAT4) (62). In contrast to the functional changes characteristic of monocyte tolerance, monocytes from children with uncomplicated malaria showed enhanced proinflammatory cytokine production and decreased phagocytosis. Transcriptional profiles of monocytes during acute malaria were in several ways distinct from those of tolerant monocytes from sepsis patients. For example, acute malaria was associated with underexpression of the chemokines CCL2 and CCL7; no change in expression of proinflammatory cytokines, such as $I L 1 B$ and IL6; and underexpression of the transcription factors HIF1A and STAT4. It is important to take into account the underlying health status and previous exposures of these patients when analyzing differences between acute disease and recovery. Notably, monocyte expression of a number of proinflammatory genes (including $I L 1 B, I L 6$, and $T N F$ ) was not different between acute malaria and recovery, though these genes were significantly overexpressed at both time points when compared with healthy malaria-naive controls. Taken together, these results suggest that, during uncomplicated malaria infection in children from endemic areas, monocytes undergo modulations of both proinflammatory and antiinflammatory functions, with a priming effect on innate immune responses that is observed several weeks after clinical recovery.

In contrast to tolerant monocytes, primed (or "trained") monocytes display enhanced proinflammatory cytokine production when challenged with a secondary stimulus (63). Our results are consistent with recent reports that demonstrated evidence for a priming effect on the innate immune response during $P$. falciparum infection. Using an experimental human malaria model, McCall et al. showed primed TLR4 and TLR2/TLR1 responses during subpatent blood-stage infection that then normalized after curative treatment (these in vivo findings were supported by an in vitro model of PBMC priming by $P$. falciparum antigens) (64). Franklin et al. demonstrated primed innate responses (to several TLR ligands, including TLR4 and TLR2) of PBMCs from Brazilian adults with uncomplicated falciparum malaria, with more robust proinflammatory cytokine responses than that observed in the experimental malaria volunteers (65). As with the volunteers, TLR responses in the Brazilian adults reversed after treatment. In our cohort, we did not observe a reversal in enhanced TLR responses after treatment, which may be due to differences in age, duration of infection, and transmission intensity. Frequent episodes of clinical malaria (among other potential infectious and environmental stimuli) may have a priming/training effect on monocytes that persists for several weeks in these children. Increased extracellular heme and proinflammatory cytokines, such as IP-10, associated with mouse malaria models and human P. falciparum infections have also been reported to increase expression of TLRs, such as TLR4, on the surface of monocyte/macrophages and circulating endothelial progenitor cells (66).

A limitation of our study is the potential influence that unmeasured confounders, including infectious, genetic, and environmental factors, may have on monocyte functions. However, the study design with acute malaria and matching recovery samples allowed each participant to serve as his/her own control and thus strengthens support for our conclusions that $P$. falciparum infection leads to modulations of monocyte functions. Another limitation is the absence of additional longitudinal samples (including those obtained prior to acute malaria episodes) that could provide more definitive data regarding the priming effect that acute malaria has on monocyte TLR responsiveness. Future prospective studies incorporating further longitudinal measurements, along with detailed measures of exposure to malaria as well as other infections, will provide crucial information regarding the relationship between innate immune responses and clinical immunity to malaria.

Malaria-induced modulation of monocyte functions likely has several important implications for the host. A general impairment in monocyte phagocytosis may contribute to increased susceptibility to invasive bacterial infections, a known complication of malaria in children (67-69). Impairment in neutrophil killing of nontyphoidal Salmonella has been observed following uncomplicated falciparum malaria in African children (51), but this impairment is related to defects in oxidative killing due to malaria-induced changes in heme-oxygenase 1 expression during myeloid cell development and not to phagocytosis. 
Priming of monocytes could be deleterious to the host if the systemic inflammatory response to subsequent TLR stimulation (by malaria or other pathogens) proceeds unchecked, driving the pathogenesis of severe infection (70). On the other hand, enhanced innate immune responses might aid in control of parasitemia, provide nonspecific antimicrobial protection, and augment vaccine responsiveness (63). Repeated activation of innate immune responses by recurring $P$. falciparum infections may play a key role in developing and maintaining clinical immunity to malaria. Further study is needed to fully define how modulated monocyte functions effect disease severity and the acquisition of antimalarial immunity in children from endemic areas.

\section{Methods}

Study site and study participants. The study was conducted at the Chulaimbo Sub-District Hospital outpatient clinic in Kisumu County, Kenya. The clinic is located approximately 10 kilometers from the research laboratory at the Kenya Medical Research Institute research station in Kisian. Malaria transmission in this area is perennially high, with peaks coinciding with seasonal rains in May-July and October-November $(31,71)$. The study population was almost exclusively of the Luo ethnicity. P. falciparum is the primary malaria parasite species (72).

Participants were enrolled in this observational cohort between June 2013 and April 2016; they included children aged 1-10 years with acute uncomplicated malaria diagnosed by light microscopy, healthy asymptomatic children aged 1-10 years, and healthy asymptomatic adults aged 18 years or older residing in location-matched areas. All cases of uncomplicated malaria were febrile (temperature $\geq 37.5^{\circ} \mathrm{C}$ ) and without signs or symptoms of severe malaria. All healthy children and adult community controls were asymptomatic and afebrile at enrollment and denied any episodes of febrile malaria or receipt of antimalarials in the 2 months preceding enrollment. Thick and thin blood smears were prepared from all blood samples. A slide was deemed negative when no parasites were seen after counting microscopic fields containing at least 200 leukocytes. The number of parasites per $\mu 1$ of blood was calculated based on each participant's total leukocyte count. Children with acute uncomplicated malaria were treated with artemether-lumefantrine per Kenyan Ministry of Health guidelines and followed up at 2 and 6 weeks. At 2 weeks, finger prick blood samples were obtained for blood smear to ensure parasite clearance. Venous blood samples were obtained from cases with acute malaria at the time of presentation (before administration of the first dose of artemether-lumefantrine) and 6 weeks following treatment and from healthy controls at enrollment.

Malaria diagnostics and related laboratory tests. Submicroscopic blood-stage infections were detected by conventional PCR of DNA samples from 6-week recovery visits and healthy control enrollment visits. A nested PCR assay targeting $P$. falciparum-specific small subunit ribosomal RNA genes was performed as previously described; the limit of detection for the assay is 6 parasites/ $\mu$ l blood (73). For cases of acute uncomplicated malaria, PfHRP2 levels in the plasma were determined using a commercial ELISA kit according to the manufacturer's instructions (Malaria Ag CELISA, Cellabs Pty. Ltd.). Extracellular heme levels were measured in plasma samples using a colorimetric assay that measures hemin, an oxidized form of heme (Biovision). The lower limit of detection was $1.03 \mu \mathrm{M}$. Blood leukocyte differentials and total leukocyte counts and routine hematological variables were measured using an automated cell counting machine (Beckman Coulter).

Indicators of systemic inflammation. We measured levels of 16 cytokines and acute-phase reactants in plasma samples from 60 children with acute malaria, the same 60 children 6 weeks following treatment, and 40 healthy child controls. All plasma samples were assayed immediately after initial thawing. A multiplexed bead-based immunoassay was used to simultaneously measure IFN- $\gamma$, TNF, IP-10, IL-10, IL-6, IL-7, IL-9, IL-17, IL-12p40, IL-12p70, IL-4, sTNF-R1, and sTNF-R2 (Millipore). Levels of CRP, soluble BAFF, and soluble CD14 were determined using commercial ELISA kits (R\&D Systems).

Monocyte subset phenotypes by flow cytometry. PBMCs were separated by Ficoll-Hypaque density gradient centrifugation and cryopreserved as previously described (74). Cryopreservation did not have a significant effect on proportions of monocyte subsets (Supplemental Figure 6), as has been shown by others previously (75). PBMCs were gently thawed and resuspended at a concentration of $10^{6}$ cells $/ \mathrm{ml}$ in RPMI-1640 (GIBCO). LIVE/DEAD Fixable Violet stain was used to assess cell viability (ThermoFisher Scientific). PBMCs were directly stained for 20 minutes at $4^{\circ} \mathrm{C}$ with a combination of fluorochrome-conjugated monoclonal antibodies, including Alexa Fluor 700-labeled anti-CD14 (clone 61D3; eBioscience), APC-Cy 7-labeled anti-CD16 (clone 3G8; BioLegend), FITC-labeled anti-CD282 TLR2 (clone TL2.1; BioLegend), PE-labeled anti-CD284 TLR4 (clone HTA125; BioLegend), PerCP-labeled anti-CD36 (clone AC106; Miltenyi Biotec), 
PE-labeled anti-CD257 BAFF (clone T7-241; BioLegend), APC-labeled anti-CD274 PD-L1 (clone 29E.2A3; BioLegend), and PE-CF594-labeled anti-CD86 (clone 2331; BD Biosciences). The stained cells were washed twice, centrifuged at $320 \mathrm{~g}$ for 10 minutes, resuspended in $100 \mu 1$ of fixing solution ( $2 \%$ paraformaldehyde kept on ice), and subjected to flow analysis using an LSRII flow cytometer (BD Biosciences). FlowJo software (Tree Star) was used for data analysis. The gating strategy put forth by the Nomenclature Committee of the International Union of Immunological Societies was used to determine proportions of monocyte subsets (classical CD $14^{++} \mathrm{CD} 16^{-}$, intermediate $\mathrm{CD} 14^{++} \mathrm{CD} 16^{+}$, and nonclassical $\mathrm{CD} 14^{+} \mathrm{CD} 16^{++}$) (21). Integrated mean fluorescence intensity values were calculated for each cell surface marker by multiplying the geometric mean fluorescence by the frequency of the parent population.

Opsonic and nonopsonic phagocytosis assays. Asexual-stage $P$. falciparum parasite cultures were maintained in group O human erythrocytes at 4\% hematocrit in RPMI-1640-HEPES medium supplemented with 5\% heat-inactivated group $\mathrm{AB}$ human serum and $5 \%$ Albumax (GIBCO), at $37^{\circ} \mathrm{C}$ with $5 \% \mathrm{CO}_{2}, 5 \% \mathrm{O}_{2}$, and $90 \% \mathrm{~N}_{2}$. Parasite cultures were enriched for knob-expressing strains using $0.75 \%$ gelatin sedimentation every 2 weeks. Cultures were checked for Mycoplasma contamination every 2-4 weeks using PCR (Sigma-Aldrich). IEs at the trophozoite stage were concentrated to $>75 \%$ purity by magnetic separation using MACS LS Columns (Miltenyi Biotec). IEs were labeled with $50 \mu \mathrm{M}$ CFSE (CellTrace CFSE, ThermoFisher Scientific) at $1 \times 10^{8}$ cells $/ \mathrm{ml}$ for 10 minutes at $37^{\circ} \mathrm{C}$. CFSE-labeled IEs were opsonized with heat-inactivated plasma (at a final plasma concentration of 1:10) for 1 hour and then washed in RPMI-1640. Plasma from malaria-naive North Americans was used as a negative control, and pooled plasma from 8 malaria-immune Kenyan adults was used as a positive control. These assays utilized IEs from the P. falciparum isolate Ghana14, described in this study. The Ghana14 isolate originated from the peripheral blood of a child with severe malaria returning to the USA from Ghana (year 2014) and was adapted to in vitro culture over several weeks. We used the Ghana14 isolate because experiments comparing phagocytosis by North American monocytes of the widely used reference isolate 3D7 versus Ghana14 showed the same patterns among the subsets but greater magnitude with Ghana14 (Supplemental Figure 7).

Opsonized CFSE-labeled IEs (concentration $10^{8} \mathrm{cells} / \mathrm{ml}$ ) were incubated with thawed cryopreserved PBMCs (concentration $2 \times 10^{6}$ cells $/ \mathrm{ml}$ ) at a 10:1 ratio of IE/PBMCs in cell culture medium (RPMI-1640 with $2 \mathrm{mM}$ L-glutamine, 10\% FBS, $10 \mathrm{mM}$ HEPES, $1 \mathrm{mM}$ sodium pyruvate, $4.5 \mathrm{~g} / 1$ glucose, $1.5 \mathrm{~g} / 1$ sodium bicarbonate, and $0.05 \mathrm{mM}$ 2-ME). The cells were incubated in polypropylene tubes for 40 minutes at $37^{\circ} \mathrm{C}$, in $5 \% \mathrm{CO}_{2}$, on an orbital shaker. After the phagocytosis incubation, the cells were placed on ice and washed in PBS, and LIVE/DEAD Fixable Violet stain was used to assess cell viability. PBMCs were directly stained for 20 minutes at $4^{\circ} \mathrm{C}$ with Alexa Fluor 700-labeled anti-CD14 (clone 61D3; eBioscience) and APC-Cy 7-labeled anti-CD16 (clone 3G8; BioLegend). Erythrocytes were lysed and leukocytes were fixed using 1-Step Fix/Lyse Solution (BioLegend). Cells were subjected to flow analysis using an LSRII flow cytometer (BD Biosciences). The percentage of monocytes within a population that had phagocytosed IEs was calculated according the CFSE fluorescence. A universal gate for CFSE positivity was set according to the signal from lysed IEs (all positive for CFSE) and from lymphocytes (all negative for CFSE).

Phagocytosis assays measured phagocytic function of monocyte subsets in PBMC samples from 12 children with acute malaria and their matching 6-week recovery samples (a single North American PBMC donor was run alongside each assay as a control). Six of the twelve participants were P. falciparum PCR negative at their 6-week recovery visits. The remaining 6 participants had asymptomatic parasitemia at their 6-week recovery visits ( 3 were blood smear positive and 3 were $P$. falciparum PCR positive and blood smear negative).

Antibodies to VSAs. Anti-VSA IgG antibodies were measured by flow cytometry as previously described with minor modifications (76). The Ghana14 P. falciparum isolate was used. Parasites were grown in group O erythrocytes, synchronized, harvested at the late trophozoite stage, and cryopreserved. Anti-VSA IgG antibodies were measured to assess the appropriateness of control plasma used in phagocytosis assays; VSA assays were performed on plasma from 24 children with acute malaria and their matching 6-week recovery samples, pooled plasma from 8 malaria-immune Kenyan adults (positive control for phagocytosis), and plasma from 2 malaria-naive North Americans (negative control for phagocytosis).

Monocyte cytokine production. Monocyte cytokine production was analyzed using freshly isolated monocytes from children presenting with acute malaria and 6 weeks following treatment ( $n=8$ pairs). Freshly isolated monocytes from healthy malaria-naive North American adults were used as positive controls $(n=4)$. Monocytes were isolated from whole blood via negative selection with the RosetteSep 
Human Monocyte Enrichment Cocktail (Stemcell Technologies, 15068). To minimize platelet contamination, the top plasma layer was removed prior to collecting monocytes at the density gradient medium/ plasma interface. Cells were suspended in cell culture medium (RPMI-1640 with $2 \mathrm{mM} \mathrm{L-glutamine,} \mathrm{10 \%}$ FBS, $10 \mathrm{mM}$ HEPES, $1 \mathrm{mM}$ sodium pyruvate, $4.5 \mathrm{~g} / 1$ glucose, $1.5 \mathrm{~g} / 1$ sodium bicarbonate, and $0.05 \mathrm{mM}$ $2-\mathrm{ME}$ ) and placed in 96-well polypropylene plates at $5 \times 10^{4}$ cells per well (concentration $5 \times 10^{5} \mathrm{cells} / \mathrm{ml}$ ). Cells were stimulated with $10 \mathrm{ng} / \mathrm{ml} \mathrm{LPS}$ (Sigma-Aldrich) and $100 \mathrm{ng} / \mathrm{ml} \mathrm{P} 3 \mathrm{C}$ (Invivogen) and compared with a media-alone control; each condition was performed in duplicate. Cells were cultured for 18 hours at $37^{\circ} \mathrm{C}$, in $5 \% \mathrm{CO}_{2}$, on an orbital shaker. Supernatants were harvested and stored at $-80^{\circ} \mathrm{C}$; all samples were assayed immediately after initial thawing. A multiplex magnetic bead-based immunoassay was used to simultaneously measure concentrations of IL-1 $\beta$, IL-6, IL-8, IL-10, IL-12p40, and TNF (Millipore) in the culture supernatants and plasma.

Targeted digital RNA sequencing. Monocyte inflammatory gene expression profiles were analyzed in samples from 6 children with acute uncomplicated malaria, their matching 6-week recovery samples, and samples from 5 malaria-naive North American adults. Whole-blood DNA samples from all 6 children at 6-week recovery visits were $P$. falciparum PCR negative. Monocytes were isolated from cryopreserved PBMCs via negative selection over a magnetic column using a Pan Monocyte Isolation Kit that enriches for all three monocyte subsets (Miltenyi Biotec, 130-096-537). Cells were lysed in RNAprotect Cell Reagent (Qiagen), and total RNA was prepared using the RNeasy kit (Qiagen) as per the manufacturer's instructions. Total RNA integrity was assessed by an Agilent 2100 Bioanalyzer, and the RNA integrity number was calculated ( $\geq 8.5$ for all samples). Targeted digital RNA sequencing was performed using a customized QIAseq Targeted RNA Panel (Human Inflammation and Immunity Transcriptome Panel) by the manufacturer (Qiagen).

To determine whether cryopreservation had a significant effect on monocyte transcript levels, we compared gene expression profiles of cryopreserved versus freshly isolated monocytes from the same North American control at the same time point. There were negligible differences in transcript levels between cryopreserved and freshly isolated monocytes $(r=0.9762, P<0.0001$, Supplemental Figure 8).

Statistics. Read counts per gene per sample from the QIAseq output were used as input for DESeq2 (77) differential gene expression analysis to identify significantly upregulated and downregulated genes for three different comparisons (Supplemental Table 5): (a) children with acute uncomplicated malaria $(n=6)$ versus their paired 6-week recovery samples $(n=6)$, using a paired sample design for more robust statistical power - with malaria, 51 genes were overexpressed and 74 were underexpressed; (b) children with acute uncomplicated malaria $(n=6)$ versus healthy malaria-naive North American adults $(n=5)$ - with malaria, 114 genes were overexpressed and 86 were underexpressed; and (c) children 6 weeks after recovery from uncomplicated malaria $(n=6)$ versus healthy malaria-naive North American adults $(n=5)$ - at 6-week recovery, 124 genes were overexpressed and 95 were underexpressed.

For each comparison, default settings were used (with a paired design for comparison [a]), and a $P=0.05$ FDR population-corrected value for significance was required for a gene to be called differentially expressed. Differentially expressed genes (separately for upregulated and downregulated genes, in each comparison) were used as input to the WebGestalt (78) functional enrichment analysis, using default settings and all 508 genes on the QIAseq Human Inflammation and Immunity Transcriptome Panel as a background set for testing. WebGestalt identifies enriched pathways, genes within the enriched pathways, and provides images of pathways maps.

For the remainder of the assays, statistical analyses were performed as follows. Wilcoxon matched-pairs rank test was used to compare acute malaria to matching 6-week recovery samples. Kruskal-Wallis test with Dunn's test for multiple comparisons was used to compare continuous variables among unmatched groups. Fisher's exact test was used to compare categorical variables between acute malaria cases and healthy child controls. Friedman test with Dunn's test for multiple comparisons was used to compare phagocytic activity among the three monocyte subsets. Spearman correlation was used to examine the relationship between any two variables. Differences were considered significant at $P<0.05$. Graphs were constructed and statistical analyses were performed using Prism software (version 7.0; GraphPad).

Study approval. Informed consent was obtained from all participants or their guardians in the appropriate local language (Luo). Ethical approval was obtained from the Institutional Review Board of University Hospitals Cleveland Medical Center, Cleveland, Ohio, USA (06-11-22), and from the Kenya Medical Research Institute Ethical Review Committee (SSC 2207). 


\section{Author contributions}

KRD designed, performed, and analyzed all experiments and drafted the manuscript. PE designed and performed all experiments. JV and PSO developed and coordinated the cohort study and collected participant samples. BAR and MM analyzed gene expression experiments and edited the manuscript. JWK designed and coordinated the project and cohort study, evaluated the data, provided funding for all experiments, and prepared the manuscript. AED designed and coordinated the project and cohort study, evaluated the data, and prepared the manuscript. All authors reviewed and approved the manuscript.

\section{Acknowledgments}

We thank all the parents and guardians for giving their consent and all study participants for their cooperation. We thank all members of the study and field teams at Chulaimbo hospital and the Kisian research station. We are grateful to C. Dechavanne for her critical reading of the manuscript. Support for this work was provided by the NIH/National Institute of Allergy and Infectious Diseases (R01AI095192-05 to JWK), the Burroughs Wellcome Fund/American Society of Tropical Medicine and Hygiene (to KRD), and Rainbow Babies \& Children's Foundation (to KRD).

Address correspondence to: Arlene E. Dent, Center for Global Health \& Diseases, Case Western Reserve University, 10900 Euclid Avenue LC: 4983, Cleveland, Ohio 44106, USA. Phone: 216.386.6005; Email: aed9@case.edu.

1. World Malaria Report 2015. World Health Organization. http://www.who.int/malaria/publications/world-malaria-report-2015/ report/en/. Accessed August 24, 2017.

2. Imbert P, et al. Severe falciparum malaria in children: a comparative study of 1990 and 2000 WHO criteria for clinical presentation, prognosis and intensive care in Dakar, Senegal. Trans R Soc Trop Med Hyg. 2002;96(3):278-281.

3. Reyburn $\mathrm{H}$, et al. Association of transmission intensity and age with clinical manifestations and case fatality of severe Plasmodium falciparum malaria. JAMA. 2005;293(12):1461-1470

4. Kendjo E, et al. Mortality patterns and site heterogeneity of severe malaria in African children. PLoS One. 2013;8(3):e58686.

5. Langhorne J, Ndungu FM, Sponaas AM, Marsh K. Immunity to malaria: more questions than answers. Nat Immunol. 2008;9(7):725-732.

6. Chua CL, Brown G, Hamilton JA, Rogerson S, Boeuf P. Monocytes and macrophages in malaria: protection or pathology? Trends Parasitol. 2013;29(1):26-34.

7. Ayi K, Patel SN, Serghides L, Smith TG, Kain KC. Nonopsonic phagocytosis of erythrocytes infected with ring-stage Plasmodium falciparum. Infect Immun. 2005;73(4):2559-2563.

8. Tebo AE, Kremsner PG, Luty AJ. Fcgamma receptor-mediated phagocytosis of Plasmodium falciparum-infected erythrocytes in vitro. Clin Exp Immunol. 2002;130(2):300-306.

9. Silver KL, Higgins SJ, McDonald CR, Kain KC. Complement driven innate immune response to malaria: fuelling severe malarial diseases. Cell Microbiol. 2010;12(8):1036-1045.

10. McGilvray ID, Serghides L, Kapus A, Rotstein OD, Kain KC. Nonopsonic monocyte/macrophage phagocytosis of Plasmodium falciparum-parasitized erythrocytes: a role for CD36 in malarial clearance. Blood. 2000;96(9):3231-3240.

11. Ruangirachuporn W, et al. Ultrastructural analysis of fresh Plasmodium falciparum-infected erythrocytes and their cytoadherence to human leukocytes. Am J Trop Med Hyg. 1992;46(5):511-519.

12. Krishnegowda G, et al. Induction of proinflammatory responses in macrophages by the glycosylphosphatidylinositols of Plasmodium falciparum: cell signaling receptors, glycosylphosphatidylinositol (GPI) structural requirement, and regulation of GPI activity. J Biol Chem. 2005;280(9):8606-8616.

13. Figueiredo RT, et al. Characterization of heme as activator of Toll-like receptor 4. J Biol Chem. 2007;282(28):20221-20229.

14. Gowda DC. TLR-mediated cell signaling by malaria GPIs. Trends Parasitol. 2007;23(12):596-604.

15. Ropert C, Franklin BS, Gazzinelli RT. Role of TLRs/MyD88 in host resistance and pathogenesis during protozoan infection: lessons from malaria. Semin Immunopathol. 2008;30(1):41-51.

16. Gazzinelli RT, Denkers EY. Protozoan encounters with Toll-like receptor signalling pathways: implications for host parasitism. Nat Rev Immunol. 2006;6(12):895-906.

17. Butler NS, et al. Therapeutic blockade of PD-L1 and LAG-3 rapidly clears established blood-stage Plasmodium infection. Nat Immunol. 2011;13(2):188-195.

18. Scholzen A, et al. BAFF and BAFF receptor levels correlate with B cell subset activation and redistribution in controlled human malaria infection. J Immunol. 2014;192(8):3719-3729.

19. Clark IA, Budd AC, Alleva LM, Cowden WB. Human malarial disease: a consequence of inflammatory cytokine release. Malar J. 2006;5:85.

20. Perkins DJ, Were T, Davenport GC, Kempaiah P, Hittner JB, Ong'echa JM. Severe malarial anemia: innate immunity and pathogenesis. Int J Biol Sci. 2011;7(9):1427-1442.

21. Ziegler-Heitbrock L, et al. Nomenclature of monocytes and dendritic cells in blood. Blood. 2010;116(16):e74-e80.

22. Sugimoto C, et al. Differentiation kinetics of blood monocytes and dendritic cells in macaques: insights to understanding human myeloid cell development. J Immunol. 2015;195(4):1774-1781. 
23. Strauss-Ayali D, Conrad SM, Mosser DM. Monocyte subpopulations and their differentiation patterns during infection. J Leukoc Biol. 2007;82(2):244-252.

24. Wong KL, Yeap WH, Tai JJ, Ong SM, Dang TM, Wong SC. The three human monocyte subsets: implications for health and disease. Immunol Res. 2012;53(1-3):41-57.

25. Jaworowski A, et al. CD16+ monocyte subset preferentially harbors HIV-1 and is expanded in pregnant Malawian women with Plasmodium falciparum malaria and HIV-1 infection. J Infect Dis. 2007;196(1):38-42.

26. Chimma $\mathrm{P}$, et al. A distinct peripheral blood monocyte phenotype is associated with parasite inhibitory activity in acute uncomplicated Plasmodium falciparum malaria. PLoS Pathog. 2009;5(10):e1000631.

27. Ogonda LA, Orago AS, Otieno MF, Adhiambo C, Otieno W, Stoute JA. The levels of CD16/Fc gamma receptor IIIA on CD14+ CD16+ monocytes are higher in children with severe Plasmodium falciparum anemia than in children with cerebral or uncomplicated malaria. Infect Immun. 2010;78(5):2173-2181.

28. Antonelli LR, et al. The CD14+CD16+ inflammatory monocyte subset displays increased mitochondrial activity and effector function during acute Plasmodium vivax malaria. PLoS Pathog. 2014;10(9):e1004393.

29. Zhou J, et al. CD14(hi)CD16+ monocytes phagocytose antibody-opsonised Plasmodium falciparum infected erythrocytes more efficiently than other monocyte subsets, and require CD16 and complement to do so. BMC Med. 2015;13:154.

30. Zhou G, Lee MC, Githeko AK, Atieli HE, Yan G. insecticide-treated net campaign and malaria transmission in Western Kenya: 2003-2015. Front Public Health. 2016;4:153.

31. Zhou G, et al. Changing patterns of malaria epidemiology between 2002 and 2010 in Western Kenya: the fall and rise of malaria. PLoS ONE. 2011;6(5):e20318.

32. Rubach MP, et al. Plasma Plasmodium falciparum histidine-rich protein-2 concentrations are associated with malaria severity and mortality in Tanzanian children. PLoS One. 2012;7(5):e35985.

33. Zheng J, et al. An unbalanced PD-L1/CD86 ratio in CD14(++)CD16(+) monocytes is correlated with HCV viremia during chronic HCV infection. Cell Mol Immunol. 2014;11(3):294-304.

34. Abdalla AE, Li Q, Xie L, Xie J. Biology of IL-27 and its role in the host immunity against Mycobacterium tuberculosis. Int J Biol Sci. 2015;11(2):168-175.

35. Collison LW, et al. The inhibitory cytokine IL-35 contributes to regulatory T-cell function. Nature. 2007;450(7169):566-569.

36. Li X, et al. IL-35 is a novel responsive anti-inflammatory cytokine--a new system of categorizing anti-inflammatory cytokines. PLoS One. 2012;7(3):e33628.

37. Wong KL, et al. Gene expression profiling reveals the defining features of the classical, intermediate, and nonclassical human monocyte subsets. Blood. 2011;118(5):e16-e31.

38. Patel AA, et al. The fate and lifespan of human monocyte subsets in steady state and systemic inflammation. J Exp Med. 2017;214(7):1913-1923.

39. Kratofil RM, Kubes P, Deniset JF. Monocyte conversion during inflammation and injury. Arterioscler Thromb Vasc Biol. 2017;37(1):35-42.

40. Luty AJ, et al. Low interleukin-12 activity in severe Plasmodium falciparum malaria. Infect Immun. 2000;68(7):3909-3915.

41. Weinberg JB, et al. Monocyte polarization in children with falciparum malaria: relationship to nitric oxide insufficiency and disease severity. Sci Rep. 2016;6:29151.

42. Cambos M, et al. The IL-12p70/IL-10 interplay is differentially regulated by free heme and hemozoin in murine bone-marrow-derived macrophages. Int J Parasitol. 2010;40(9):1003-1012.

43. Fontana MF, et al. A novel model of asymptomatic plasmodium parasitemia that recapitulates elements of the human immune response to chronic infection. PLoS One. 2016;11(9):e0162132.

44. de Mast $\mathrm{Q}$, et al. Is asymptomatic malaria really asymptomatic? Hematological, vascular and inflammatory effects of asymptomatic malaria parasitemia. J Infect. 2015;71(5):587-596.

45. Woodberry T, et al. Low-level Plasmodium falciparum blood-stage infection causes dendritic cell apoptosis and dysfunction in healthy volunteers. J Infect Dis. 2012;206(3):333-340.

46. Nankabirwa J, Wandera B, Kiwanuka N, Staedke SG, Kamya MR, Brooker SJ. Asymptomatic Plasmodium infection and cognition among primary schoolchildren in a high malaria transmission setting in Uganda. Am J Trop Med Hyg. 2013;88(6):1102-1108.

47. Chen I, et al. "Asymptomatic" malaria: a chronic and debilitating infection that should be treated. PLoS Med. 2016;13(1):e1001942.

48. Schwarzer E, Turrini F, Ulliers D, Giribaldi G, Ginsburg H, Arese P. Impairment of macrophage functions after ingestion of Plasmodium falciparum-infected erythrocytes or isolated malarial pigment. J Exp Med. 1992;176(4):1033-1041.

49. Sarantis H, Grinstein S. Subversion of phagocytosis for pathogen survival. Cell Host Microbe. 2012;12(4):419-431.

50. Martins R, et al. Heme drives hemolysis-induced susceptibility to infection via disruption of phagocyte functions. Nat Immunol. 2016;17(12):1361-1372.

51. Cunnington AJ, Njie M, Correa S, Takem EN, Riley EM, Walther M. Prolonged neutrophil dysfunction after Plasmodium falciparum malaria is related to hemolysis and heme oxygenase-1 induction. J Immunol. 2012;189(11):5336-5346.

52. Ayi K, et al. CD47-SIRPa Interactions regulate macrophage uptake of plasmodium falciparum-infected erythrocytes and clearance of malaria in vivo. Infect Immun. 2016;84(7):2002-2011.

53. Ravetch JV, Lanier LL. Immune inhibitory receptors. Science. 2000;290(5489):84-89.

54. Munoz C, Carlet J, Fitting C, Misset B, Blériot JP, Cavaillon JM. Dysregulation of in vitro cytokine production by monocytes during sepsis. J Clin Invest. 1991;88(5):1747-1754.

55. Monneret G, et al. The anti-inflammatory response dominates after septic shock: association of low monocyte HLA-DR expression and high interleukin-10 concentration. Immunol Lett. 2004;95(2):193-198.

56. Medvedev AE, Kopydlowski KM, Vogel SN. Inhibition of lipopolysaccharide-induced signal transduction in endotoxin-tolerized mouse macrophages: dysregulation of cytokine, chemokine, and toll-like receptor 2 and 4 gene expression. J Immunol. 2000;164(11):5564-5574.

57. Foster SL, Hargreaves DC, Medzhitov R. Gene-specific control of inflammation by TLR-induced chromatin modifications. Nature. 2007;447(7147):972-978. 
58. Salomao R, Brunialti MK, Rapozo MM, Baggio-Zappia GL, Galanos C, Freudenberg M. Bacterial sensing, cell signaling, and modulation of the immune response during sepsis. Shock. 2012;38(3):227-242.

59. Biswas SK, Lopez-Collazo E. Endotoxin tolerance: new mechanisms, molecules and clinical significance. Trends Immunol. 2009;30(10):475-487.

60. del Fresno C, et al. Potent phagocytic activity with impaired antigen presentation identifying lipopolysaccharide-tolerant human monocytes: demonstration in isolated monocytes from cystic fibrosis patients. J Immunol. 2009;182(10):6494-6507.

61. Fernandes ML, Mendes ME, Brunialti MK, Salomão R. Human monocytes tolerant to LPS retain the ability to phagocytose bacteria and generate reactive oxygen species. Braz J Med Biol Res. 2010;43(9):860-868.

62. Shalova IN, et al. Human monocytes undergo functional re-programming during sepsis mediated by hypoxia-inducible factor-1 $\alpha$. Immunity. 2015;42(3):484-498.

63. Netea MG, et al. Trained immunity: A program of innate immune memory in health and disease. Science. 2016;352(6284):aaf1098.

64. McCall MB, et al. Plasmodium falciparum infection causes proinflammatory priming of human TLR responses. J Immunol. 2007;179(1):162-171.

65. Franklin BS, et al. Malaria primes the innate immune response due to interferon-gamma induced enhancement of toll-like receptor expression and function. Proc Natl Acad Sci USA. 2009;106(14):5789-5794.

66. Dickinson-Copeland CM, et al. Heme-mediated induction of CXCL10 and depletion of CD34+ progenitor cells is Toll-like receptor 4 dependent. PLoS One. 2015;10(11):e0142328

67. Berkley J, Mwarumba S, Bramham K, Lowe B, Marsh K. Bacteraemia complicating severe malaria in children. Trans R Soc Trop Med Hyg. 1999;93(3):283-286.

68. Bronzan RN, et al. Bacteremia in Malawian children with severe malaria: prevalence, etiology, HIV coinfection, and outcome J Infect Dis. 2007;195(6):895-904.

69. Berkley JA, et al. HIV infection, malnutrition, and invasive bacterial infection among children with severe malaria. Clin Infect Dis. 2009;49(3):336-343.

70. Mackintosh CL, Beeson JG, Marsh K. Clinical features and pathogenesis of severe malaria. Trends Parasitol. 2004;20(12):597-603.

71. Desai M, et al. Age-specific malaria mortality rates in the KEMRI/CDC health and demographic surveillance system in western Kenya, 2003-2010. PLoS One. 2014;9(9):e106197.

72. Munyekenye OG, Githeko AK, Zhou G, Mushinzimana E, Minakawa N, Yan G. Plasmodium falciparum spatial analysis, western Kenya highlands. Emerging Infect Dis. 2005;11(10):1571-1577.

73. Singh B, Bobogare A, Cox-Singh J, Snounou G, Abdullah MS, Rahman HA. A genus- and species-specific nested polymerase chain reaction malaria detection assay for epidemiologic studies. Am J Trop Med Hyg. 1999;60(4):687-692.

74. Fuss IJ, Kanof ME, Smith PD, Zola H. Isolation of whole mononuclear cells from peripheral blood and cord blood. Curr Protoc Immunol. 2009; Chapter 7:Unit7.1.

75. Appleby LJ, Nausch N, Midzi N, Mduluza T, Allen JE, Mutapi F. Sources of heterogeneity in human monocyte subsets. Immunol Lett. 2013;152(1):32-41.

76. Staalsoe T, Giha HA, Dodoo D, Theander TG, Hviid L. Detection of antibodies to variant antigens on Plasmodium falciparum-infected erythrocytes by flow cytometry. Cytometry. 1999;35(4):329-336.

77. Love MI, Huber W, Anders S. Moderated estimation of fold change and dispersion for RNA-seq data with DESeq2. Genome Biol. 2014;15(12):550.

78. Wang J, Duncan D, Shi Z, Zhang B. WEB-based Gene SeT AnaLysis Toolkit (WebGestalt): update 2013. Nucleic Acids Res. 2013;41(Web Server issue):W77-W83 